Pacific

Journal of

Mathematics

ON THE PRESCRIBED SCALAR CURVATURE PROBLEM ON THE THREE-DIMENSIONAL HALF SPHERE

Mohamed Ben Ayed And Hichem Chtioui 


\title{
ON THE PRESCRIBED SCALAR CURVATURE PROBLEM ON THE THREE-DIMENSIONAL HALF SPHERE
}

\author{
Mohamed Ben Ayed And Hichem Chtioui
}

\begin{abstract}
We provide a variety of classes of functions that can be realized as the scalar curvature of the standard three-dimensional half sphere with respect to some metric whose boundary mean curvature is zero. Such a problem is nontrivial, since we have to overcome topological obstructions.
\end{abstract}

\section{Introduction and Results}

In this paper we study some equations arising in differential geometry when the metric of a Riemannian manifold is conformally deformed. More precisely, given a manifold with boundary $(M, g)$ of dimension $n \geq 3$, transforming the metric $g$ into $\tilde{g}=u^{4 /(n-2)} g$, where $u$ is a smooth positive function, the scalar curvatures $R_{g}$ and $R_{\tilde{g}}$, and the mean curvatures $h_{g}$ and $h_{\tilde{g}}$ of the boundary, with respect to $g$ and $\tilde{g}$ respectively, are related by the formulas

$$
\left\{\begin{aligned}
-c_{n} \Delta_{g} u+R_{g} u & =R_{\tilde{g}} u^{(n+2) /(n-2)} & & \text { in } M, \\
\frac{2}{n-2} \frac{\partial u}{\partial v}+h_{g} u & =h_{\tilde{g}} u^{n /(n-2)} & & \text { on } \partial M,
\end{aligned}\right.
$$

where $c_{n}=4(n-1) /(n-2)$ and $v$ denotes the outward normal vector with respect to the metric $g$.

In view of $\left(P_{1}\right)$, the following problem naturally arises: given two functions $K: M \rightarrow \mathbb{R}$ and $h: \partial M \rightarrow \mathbb{R}$, does there exist a metric $\tilde{g}$ conformally equivalent to $g$ such that $R_{\tilde{g}} \equiv K$ and $h_{\tilde{g}} \equiv h$ ? From $\left(P_{1}\right)$ the problem is equivalent to finding a smooth positive solution $u$ of the equation

$$
\begin{cases}-c_{n} \Delta_{g} u+R_{g} u=K u^{(n+2) /(n-2)} & \text { in } M, \\ \frac{2}{n-2} \frac{\partial u}{\partial v}+h_{g} u=h u^{n /(n-2)} & \text { on } \partial M .\end{cases}
$$

The positivity requirement on $u$ is necessary for the metric $\tilde{g}$ to be Riemannian. Such a problem was studied in [Ambrosetti et al. 2000; Ben Ayed et al. 2002, 2005;

MSC2000: 36J60, 35J65, 58E05.

Keywords: scalar curvature problem, variational problem, lack of compactness, critical points at infinity, 3D half-sphere. 
Cherrier 1984; Chtioui and El Mehdi 2003; Djadli et al. 2003; Escobar 1996; Han and Li 2000; Li 1995a].

It is well known that the positive case is the most interesting one, that is, when the quadratic part of the associated Euler functional is positive definite. Another interesting case is when a noncompact group of conformal transformations acts on the equation, leading to topological obstructions. The half sphere represents the simplest case where such noncompactness occurs, and in this case problem $\left(P_{2}\right)$ is thus reduced to that of finding a positive solution of

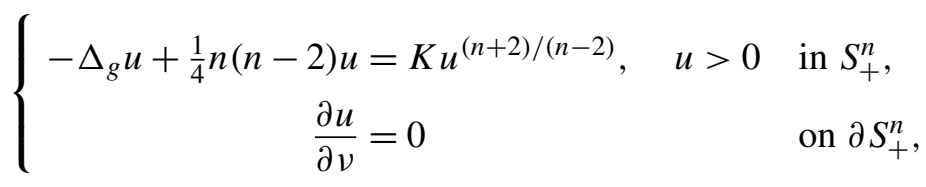

where $S_{+}^{n}=\left\{x \in \mathbb{R}^{n+1}:|x|=1, x_{n+1}>0\right\}$ and $g$ is the standard metric of $S_{+}^{n}$.

Problem (1-2) is in some sense related to the well known scalar curvature problem on $S^{n}$

$$
-\Delta_{g} u+\frac{1}{4} n(n-2) u=K u^{(n+2) /(n-2)} \quad \text { in } S^{n}
$$

to which much work has been devoted. For details, see [Bahri 1996; Bahri and Coron 1991; Ben Ayed et al. 1996; Chang et al. 1993; Chang and Yang 1991; Hebey 1990; Li 1995b; Schoen and Zhang 1996] and the references therein. As for (1-3), there are topological obstructions of Kazdan-Warner type to solving (1-2) (see [Bianchi and Pan 1999]) and so a natural question arises: under which conditions on $K$ does (1-2) have a positive solution? In this paper we consider the case of the standard three-dimensional half sphere under minimal boundary conditions. Our goal is to look for conditions on $K$ to ensure the existence of a positive solution of the problem

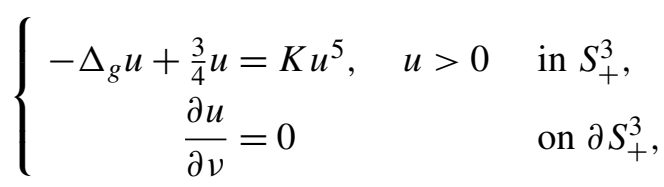

where $K$ is a $C^{3}$-function on $\overline{S_{+}^{3}}$.

We propose to study problem (1-4), using some topological and dynamical tools of the theory of critical points at infinity, see [Bahri 1989; 1996]. Our approach follows the ideas developed in [Aubin and Bahri 1997; Bahri 1996; Ben Ayed et al. 1996] where the problem of prescribing the scalar curvature on closed manifolds was studied using some algebraic topological tools. The main idea is to use the topological differences between the level sets of the function $K$ to produce a critical point of the Euler functional $J$ associated to $(1-4)$ and the main issue is under which conditions on $K$, a topological accident between the level sets of $K$ induces 
a topological accident between the level sets of $J$. Such an accident is sufficient to prove the existence of a critical point when some compactness conditions are satisfied. However our problem presents a lack of compactness due to the presence of critical points at infinity, that is, noncompact orbits for the gradient of $J$ along which $J$ is bounded and its gradient tends to zero. Therefore a careful study of such noncompact orbits is necessary, in order to take into account their contribution to the difference of topology between the level sets of $J$.

Note that Bahri [1989] observed that a new phenomenon appears for problem (1-3) in dimension $n \geq 5$ due to the fact that the self-interaction of the functions failing the Palais-Smale condition dominates the interaction of two of those functions. In the three-dimensional case, the reverse happens [Bahri and Coron 1991]. In dimension 4, we have a balance phenomenon, that is, the self-interaction and the interaction are of the same size [Ben Ayed et al. 1996].

For problem (1-2), [Djadli et al. 2003] showed that such a balance phenomenon appears in $S_{+}^{3}$. Such a result suggests that there is a dimension break between problem (1-2) and problem (1-3). Ben Ayed, El Mehdi and Ould Ahmedou [2005], proved that a dimension break is not always true. Precisely, they showed that $S_{+}^{4}$ behaves like $S^{4}$ under some conditions on the behavior of the function $K$ on the boundary $\partial S_{+}^{4}$. In this paper, we prove that $S_{+}^{3}$ behaves like $S^{3}$ and $S^{4}$ at the same time.

We also note that Yanyan Li [1995a] and Djadli, Malchiodi and Ould Ahmedou [2003] studied problem (1-4). Their approach involves a fine blow-up analysis of some subcritical approximations and the topological degree tools. In their papers they assumed that $\frac{\partial K}{\partial v}(y) \neq 0$ for each critical point $y$ of $\left.K\right|_{\partial S_{+}^{3}}$. In this work, we will give some existence results without this assumption.

For our first result, we borrow some of the ideas developed in [Ben Ayed et al. 1996] where the problem of prescribing the scalar curvature on four closed manifolds was studied using an Euler-Poincaré characteristic argument.

In order to state our results, we need to introduce some notations and the assumptions that we are using in this paper.

Let $G_{1}$ be defined by

$$
G_{1}(x, y)=(1-\cos d(x, y))^{-1 / 2}, \quad \text { for } x \neq y .
$$

Throughout this paper, we assume that $K_{1}:=\left.K\right|_{\partial S_{+}^{3}}$ has only nondegenerate critical points $y_{0}, y_{1}, \ldots, y_{s}$ such that $y_{0}$ is the absolute maximum of $K_{1}$. We set

$$
\begin{aligned}
I_{+} & =\left\{y: \nabla K_{1}(y)=0 \text { and }(\partial K / \partial v)(y)>0\right\}, \\
I_{0}^{+} & =\left\{y: \nabla K_{1}(y)=0,(\partial K / \partial v)(y)=0, \text { and }-\Delta K(y)>0\right\}, \\
\mathscr{F}_{N} & =\left\{\left(q_{1}, \ldots, q_{N}\right) \in\left(I_{+}\right)^{N}: q_{i} \neq q_{j} \text { for } i \neq j\right\}, \quad \text { with } N \in \mathbb{N}^{*} .
\end{aligned}
$$


To each $\left(q_{1}, \ldots, q_{N}\right) \in \mathscr{F}_{N}$, we associate an $N \times N$ symmetric matrix

defined by

$$
M=M\left(q_{1}, \ldots, q_{N}\right)
$$

(1-5) $\quad M_{i i}=\frac{\partial K}{\partial v}\left(q_{i}\right) \frac{1}{K\left(q_{i}\right)^{3 / 2}}, \quad M_{i j}=-\frac{4 \sqrt{2} G_{1}\left(q_{i}, q_{j}\right)}{\left(K\left(q_{i}\right) K\left(q_{j}\right)\right)^{1 / 4}} \quad$ for $i \neq j$.

We assume that, for $N \leq \operatorname{card} I_{+}$and $\left(q_{1}, \ldots, q_{N}\right) \in \mathscr{F}_{N}$,

$M\left(q_{1}, \ldots, q_{N}\right)$ is nondegenerate.

Let $\rho=\rho\left(q_{1}, \ldots, q_{N}\right)$ be the smallest eigenvalue of $M$. It has been first pointed out by Bahri [1989] (see also [Ben Ayed et al. 1996]) that when the interaction between the different bubbles is of the same order as the self-interaction, the function $\rho$ plays a fundamental role in the theory of the critical points at infinity. For problem (1-2), such a kind of phenomenon may appear when $n=3$. In addition, we assume that $\Delta K(y) \neq 0$ for each critical point $y$ where $\partial K / \partial v$ vanishes, and there exists $\bar{r}>0$ such that $-\Delta K(y) \frac{\partial K(a)}{\partial v} \geq 0$ for all $a \in B(y, \bar{r}) \cap \partial S_{+}^{3}$.

Theorem 1.1. Under assumptions $\left(A_{1}\right)$ and $\left(A_{2}\right)$, if

$$
\sum_{y \in I_{0}^{+}}(-1)^{i(y)}+\sum_{N=1}^{\operatorname{card} I_{+}} \sum_{\substack{\tau_{N}=\left(y_{j_{1}}, \ldots, y_{j_{N}}\right) \in \mathscr{F}_{N} \\ \rho\left(\tau_{N}\right)>0}}(-1)^{3 N-1-\sum_{k=1}^{N} i\left(y_{j_{k}}\right)} \neq 1
$$

then problem (1-4) has a solution. Here, $i(y)$ denotes the Morse index of $K_{1}$ at $y$.

If we assume that $(\partial K / \partial v)(y) \neq 0$ for each critical point $y$ of $K_{1}$, we obtain [Djadli et al. 2003, Theorem 1.2] and therefore $S_{+}^{3}$ behaves like $S^{4}$. However, if we assume that

$$
\frac{\partial K}{\partial v}(y) \leq 0 \text { for each critical point } y \text { of } K_{1},
$$

we observe that $S_{+}^{3}$ behaves like $S^{3}$. Indeed:

Corollary 1.2. Under assumptions $\left(A_{2}\right)$ and $\left(A_{3}\right)$, if

$$
\sum_{y \in I_{0}^{+}}(-1)^{i(y)} \neq 1
$$

then problem (1-4) has a solution.

Now, we will give another kind of existence result based on the behavior of $K$ on the boundary. For this purpose, Let $Z$ be a pseudogradient of $K_{1}=\left.K\right|_{\partial S_{+}^{3}}$, of Morse-Smale type (that is the intersections of the stable and the unstable manifolds 
of the critical points of $K_{1}$ are transverse). We assume that

$$
\begin{array}{rlrl}
i(y) & \geq 1 & \text { for } y \in I_{+} \cup I_{0}^{+}, \\
W_{s}(y) \cap W_{u}\left(y_{j}\right) & =\varnothing \quad \text { for } y_{j} \notin I_{+} \cup I_{0}^{+} .
\end{array}
$$

Set $X_{1}=\overline{\bigcup_{y \in I_{+} \cup I_{0}^{+}} W_{s}(y)}$. We assume that

$$
X_{1} \text { is not contractible }
$$

and denote by $m$ the dimension of its first nontrivial reduced homology group.

Theorem 1.3. Then, under assumptions $\left(A_{2}\right),\left(A_{4}\right)$ and $\left(A_{5}\right)$, there exists a constant $c_{0}$ independent of $K$ such that if

$$
\left|K_{1}-1\right|_{L^{\infty}\left(\partial S_{+}^{3}\right)} \leq c_{0},
$$

then problem (1-4) has a solution of an augmented Morse index $\geq m$, where $m$ is defined in $\left(A_{5}\right)$.

Now we will focus our attention on the critical point $y_{0}$ (recall that $K_{1}\left(y_{0}\right)=$ max $K_{1}$ ). Djadli, Malchiodi and Ould Ahmedou [Djadli et al. 2003] proved that problem $(1-4)$ has a solution if $y_{0}$ satisfies $(\partial K / \partial \nu)\left(y_{0}\right)<0$. Therefore, in the remainder of this paper, we will assume that $y_{0}$ satisfies $(\partial K / \partial v)\left(y_{0}\right) \geq 0$. Our first result in this direction is the following.

Theorem 1.4. Assume that $y_{0}$ satisfies

$$
\frac{\partial K}{\partial v}\left(y_{0}\right)=0 \quad \text { and } \quad-\Delta K\left(y_{0}\right)<0 .
$$

Then, under the assumption $\left(A_{2}\right)$ (the assumption is needed only to be satisfied on $\left.y_{0}\right)$, problem (1-4) has a solution $w$ under the level $2^{-2 / 3} K\left(y_{0}\right)^{-1 / 3} S$, where $S$ is the best Sobolev constant for the embedding of $H_{0}^{1}(\Omega)$ into $L^{6}(\Omega)$.

Now, to obtain another existence result, we will give some assumptions on a critical point $y_{i_{0}}$ which satisfies

$$
y_{i_{0}} \in I_{+} \cup I_{0}^{+} \text {and } K_{1}\left(y_{i_{0}}\right)=\max \left\{K_{1}(y) / y \in I_{+} \cup I_{0}^{+}-\left\{y_{0}\right\}\right\} .
$$

We let $k:=2-i\left(y_{i_{0}}\right)$ and we assume that

$\left(A_{6}\right) \quad k \geq 1$ and $W_{s}\left(y_{i_{0}}\right) \cap W_{u}(y)=\varnothing$ for each critical point $y \notin I_{+} \cup I_{0}^{+}$.

Then we have the following existence results:

Theorem 1.5. Under assumptions $\left(A_{2}\right)$ and $\left(A_{6}\right)$, if

(i) $y_{0}, y_{i_{0}} \in I_{+} \cup I_{0}^{+}$and at least one of them belongs to $I_{0}^{+}$, and

(ii) $K(y)^{-1 / 2}>K\left(y_{0}\right)^{-1 / 2}+K\left(y_{i_{0}}\right)^{-1 / 2}$ for any $y \in I_{+} \cup I_{0}^{+}-\left\{y_{0}, y_{i_{0}}\right\}$,

then (1-4) has a solution of an augmented Morse index $k$ or $k+1$. 
Theorem 1.6. Under assumption $\left(A_{6}\right)$, if

(i) $y_{0}, y_{i_{0}} \in I_{+}, M\left(y_{0}, y_{i_{0}}\right)$ is nondegenerate and $\rho\left(y_{0}, y_{i_{0}}\right)<0$, and

(ii) $K(y)^{-1 / 2}>K\left(y_{0}\right)^{-1 / 2}+K\left(y_{i_{0}}\right)^{-1 / 2}$ for any $y \in I_{+} \cup I_{0}^{+}-\left\{y_{0}, y_{i_{0}}\right\}$,

then (1-4) has a solution of an augmented Morse index $k$ or $k+1$.

Remark 1.7. Theorems 1.5 and 1.6 also hold if we replace the respective assumptions (ii) by the following assumption:

(iii) For each $y \in I_{+} \cup I_{0}^{+}-\left\{y_{i_{0}}\right\}$ such that $K(y)^{-1 / 2} \leq K\left(y_{0}\right)^{-1 / 2}+K\left(y_{i_{0}}\right)^{-1 / 2}$, we have $i(y) \notin\{2-k, 2-(k+1)\}$.

In contrast to Theorem 1.6, we now give a result (Theorem 1.8) based on a certain topological invariant for Yamabe type problems, introduced by Bahri [1996]. First we need to introduce some notation and assumptions. Assume $\rho\left(y_{0}, y_{i_{0}}\right)>0$ and define

$$
X=\overline{W_{s}\left(y_{i_{0}}\right)} \text {. }
$$

$X$ is a manifold of dimension $k$ without boundary. Let $\delta_{x}$ be the Dirac mass at $x$. We denote by $C_{y_{0}}(X)$ the set

$$
C_{y_{0}}(X)=\left\{\alpha \delta_{y_{0}}+(1-\alpha) \delta_{x}: \alpha \in[0,1], x \in X\right\} .
$$

For $\lambda$ large enough, we introduce a map $f_{\lambda}: C_{y_{0}}(X) \rightarrow \Sigma^{+}$, defined by

$$
\left(\alpha \delta_{y_{0}}+(1-\alpha) \delta_{x}\right) \mapsto \frac{\alpha K\left(y_{0}\right)^{-1 / 4} \tilde{\delta}_{\left(y_{0}, \lambda\right)}+(1-\alpha) K(x)^{-1 / 4} \tilde{\delta}_{(x, \lambda)}}{\left\|\alpha K\left(y_{0}\right)^{-1 / 4} \tilde{\delta}_{\left(y_{0}, \lambda\right)}+(1-\alpha) K(x)^{-1 / 4} \tilde{\delta}_{(x, \lambda)}\right\|},
$$

where $\Sigma^{+}$and $\tilde{\delta}_{(a, \lambda)}$ are defined in the next section. Then $C_{y_{0}}(X)$ and $f_{\lambda}\left(C_{y_{0}}(X)\right)$ are manifolds in dimension $k+1$, that is, their singularities arise in dimension $k-1$ and lower [Bahri 1996]. Observe that $C_{y_{0}}(X)$ and $f_{\lambda}\left(C_{y_{0}}(X)\right)$ are contractible while $X$ is not. For $\lambda$ large enough, we also define the intersection number $(\bmod 2)$ of $f_{\lambda}\left(C_{y_{0}}(X)\right)$ with $W_{s}\left(y_{0}, y_{i_{0}}\right)_{\infty}$

$$
\mu\left(y_{0}, y_{i_{0}}\right)=f_{\lambda}\left(C_{y_{0}}(X)\right) . W_{s}\left(y_{0}, y_{i_{0}}\right)_{\infty},
$$

where $W_{s}\left(y_{0}, y_{i_{0}}\right)_{\infty}$ is the stable manifold of the critical point at infinity $\left(y_{0}, y_{i_{0}}\right)_{\infty}$ (see Corollary 3.2 below) for a decreasing pseudogradient $V$ for $J$ which is transverse to $f_{\lambda}\left(C_{y_{0}}(X)\right)$. Thus this number is well defined (see [Milnor 1965]).

Now introduce the assumption

$$
4 K\left(y_{i_{0}}\right)<K\left(y_{0}\right)
$$

Theorem 1.8. Under assumptions $\left(A_{6}\right)$ and $\left(A_{7}\right)$, if $y_{0}, y_{i_{0}} \in I_{+}$are such that

$$
\rho\left(y_{0}, y_{i_{0}}\right)>0, \quad \text { and } \mu\left(y_{0}, y_{i_{0}}\right)=0 \text {, }
$$

then (1-4) has a solution of an augmented Morse index $k$ or $k+1$. 
The remainder of the paper is organized as follows. Section 2 sets up the variational structure and gives some useful results, while in Section 3 we characterize the critical points at infinity of the Euler functional associated to (1-4). Finally, Section 4 is devoted to the proofs of our results.

\section{Variational Structure and Preliminaries}

In this section we recall the functional setting, the variational structure of the problem and its main features. Problem (1-2) has a variational structure. The corresponding functional is

$$
J(u)=\frac{\|u\|^{2}}{\left(\int_{S_{+}^{n}} K u^{2 n /(n-2)}\right)^{(n-2) / n}}
$$

defined on $H^{1}\left(S_{+}^{n}, \mathbb{R}\right) \backslash\{0\}$ equipped with the norm

$$
\|u\|^{2}=\int_{S_{+}^{n}}|\nabla u|^{2}+\frac{1}{4} n(n-2) \int_{S_{+}^{n}} u^{2} .
$$

We denote by $\Sigma$ the unit sphere of $H^{1}\left(S_{+}^{n}, \mathbb{R}\right)$ and set

$$
\Sigma^{+}=\{u \in \Sigma: u \geq 0\} .
$$

The functional $J$ does not satisfy the Palais-Smale condition on $\Sigma^{+}$. Its failure has been studied by various authors [Brezis and Coron 1985; Lions 1985; Struwe 1984]. In order to characterize the sequences failing the Palais-Smale condition, we need to introduce some notations. For $a \in \overline{S_{+}^{n}}$ and $\lambda>0$, let

$$
\tilde{\delta}_{a, \lambda}(x)=c_{0} \frac{\lambda^{(n-2) / 2}}{\left(\lambda^{2}+1+\left(1-\lambda^{2}\right) \cos d(a, x)\right)^{(n-2) / 2}},
$$

where $d$ is the geodesic distance on $\left(S_{+}^{n}, g\right)$ and $c_{0}$ is chosen so that

$$
-\Delta \tilde{\delta}_{a, \lambda}+\frac{1}{4} n(n-2) \tilde{\delta}_{a, \lambda}=\tilde{\delta}_{a, \lambda}^{(n+2) /(n-2)} \quad \text { in } S_{+}^{n} .
$$

Observe that $\partial \tilde{\delta}_{a, \lambda} / \partial v=0$ for $a \in \partial S_{+}^{3}$. However, $\partial \tilde{\delta}_{a, \lambda} / \partial v \neq 0$ for $a \notin \partial S_{+}^{3}$. Thus, we need to introduce another function $\varphi_{a, \lambda}$ which satisfies

$$
\left\{\begin{aligned}
-\Delta \varphi_{(a, \lambda)}+\frac{1}{4} n(n-2) \varphi_{(a, \lambda)} & =\tilde{\delta}_{a, \lambda}^{(n+2) /(n-2)} & & \text { in } S_{+}^{n}, \\
\frac{\partial \varphi_{(a, \lambda)}}{\partial v} & =0 & & \text { on } \partial S_{+}^{n} .
\end{aligned}\right.
$$

For $\varepsilon>0$ and $p \in \mathbb{N}^{*}$, define

$$
V(p, \varepsilon)
$$


as the set of $u \in \Sigma^{+}$such that there exist $a_{1}, \ldots, a_{p} \in \overline{S_{+}^{n}}, \lambda_{1}, \ldots, \lambda_{p}>0$, and $\alpha_{1}, \ldots, \alpha_{p}>0$ with the following property: if we set $\tilde{\delta}_{i}=\tilde{\delta}_{a_{i}, \lambda_{i}}, d_{i}=d\left(a_{i}, \partial S_{+}^{n}\right)$, and

$$
\varepsilon_{i j}=\left(\lambda_{i} / \lambda_{j}+\lambda_{j} / \lambda_{i}+\frac{1}{2} \lambda_{i} \lambda_{j}\left(1-\cos d\left(a_{i}, a_{j}\right)\right)\right)^{(2-n) / 2},
$$

then $\lambda_{i}>\varepsilon^{-1}, \varepsilon_{i j}<\varepsilon$, and $\lambda_{i} d_{i} \notin\left[\varepsilon, \varepsilon^{-1}\right]$,

$$
\left\|u-\sum_{i=1}^{p} \alpha_{i} \tilde{\delta}_{i}\right\|<\varepsilon \quad \text { and } \quad\left|\frac{\alpha_{i}^{4 /(n-2)} K\left(a_{i}\right)}{\alpha_{j}^{4 /(n-2)} K\left(a_{j}\right)}-1\right|<\varepsilon .
$$

The failure of the Palais-Smale condition can be described as follows:

Proposition 2.1 [Brezis and Coron 1985; Lions 1985; Struwe 1984]. Suppose that $J$ has no critical point in $\Sigma^{+}$and let $\left(u_{k}\right) \in \Sigma^{+}$be a sequence such that $J\left(u_{k}\right)$ is bounded and $\nabla J\left(u_{k}\right) \rightarrow 0$. Then there is an integer $p \in \mathbb{N}^{*}$, a sequence $\varepsilon_{k}>0$ (with $\varepsilon_{k} \rightarrow 0$ ) and an extracted subsequence of $u_{k}$, again denoted $\left(u_{k}\right)$, such that $u_{k} \in V\left(p, \varepsilon_{k}\right)$.

If a function $u$ belongs to $V(p, \varepsilon)$, we assume, for simplicity, that $\lambda_{i} d_{i}<\varepsilon$ for $i \leq q$, and $\lambda_{i} d_{i}>\varepsilon^{-1}$ for $i>q$. We consider the following minimization problem for $u \in V(p, \varepsilon)$ with $\varepsilon$ small:

$$
\begin{array}{r}
\min \left\{\left\|u-\sum_{i=1}^{q} \alpha_{i} \tilde{\delta}_{\left(a_{i}, \lambda_{i}\right)}-\sum_{i=q+1}^{p} \alpha_{i} \varphi_{\left(b_{i}, \lambda_{i}\right)}\right\|: \alpha_{i}>0, \lambda_{i}>0\right. \\
\left.a_{i} \in \partial S_{+}^{3}, b_{i} \in S_{+}^{3}\right\} .
\end{array}
$$

The next proposition defines a parametrization of the set $V(p, \varepsilon)$, and follows from corresponding statements in [Bahri 1989; Bahri and Coron 1988; Rey 1997].

Proposition 2.2. For any $p \in \mathbb{N}^{*}$, there exists $\varepsilon_{p}>0$ such that if $\varepsilon<\varepsilon_{p}$ and $u \in$ $V(p, \varepsilon)$, the minimization problem (2-2) has a unique solution (up to permutation). In particular, we can write $u \in V(p, \varepsilon)$ as

$$
u=\sum_{i=1}^{q} \bar{\alpha}_{i} \tilde{\delta}_{\left(\bar{a}_{i}, \bar{\lambda}_{i}\right)}+\sum_{i=q+1}^{p} \bar{\alpha}_{i} \varphi_{\left(\bar{a}_{i}, \bar{\lambda}_{i}\right)}+v,
$$

where $\left(\bar{\alpha}_{1}, \ldots, \bar{\alpha}_{p}, \bar{a}_{1}, \ldots, \bar{a}_{p}, \bar{\lambda}_{1}, \ldots, \bar{\lambda}_{p}\right)$ is the solution of $(2-2)$ and $v \in H^{1}\left(S_{+}^{n}\right)$ is such that

$\left(V_{0}\right) \quad\|v\| \leq \varepsilon, \quad(v, \psi)=0: \psi \in\left\{\tilde{\delta}_{i}, \frac{\partial \tilde{\delta}_{i}}{\partial \lambda_{i}}, \frac{\partial \tilde{\delta}_{i}}{\partial a_{i}}, \varphi_{j}, \frac{\partial \varphi_{j}}{\partial \lambda_{j}}, \frac{\partial \varphi_{j}}{\partial a_{j}}: i \leq q, j>q\right\}$.

To proof of the next proposition follows, with minor modification, its analogues in [Bahri 1989] (see also [Rey 1997]). 
Proposition 2.3. There exists a $C^{1}$ map which, to each

$$
\left(\alpha_{1}, \ldots, \alpha_{p}, a_{1}, \ldots, a_{p}, \lambda_{1}, \ldots, \lambda_{p}\right)
$$

such that

$$
\sum_{i=1}^{p} \alpha_{i} \tilde{\delta}_{i}+\sum_{i=q+1}^{p} \alpha_{i} \varphi_{i} \in V(p, \varepsilon)
$$

with small $\varepsilon$, associates a function $\bar{v}=\bar{v}_{\left(\alpha_{i}, a_{i}, \lambda_{i}\right)}$ satisfying

$$
J\left(\sum_{i=1}^{q} \alpha_{i} \tilde{\delta}_{i}+\sum_{i=q+1}^{p} \alpha_{i} \varphi_{i}+\bar{v}\right)=\min _{v \text { satisfying }\left(V_{0}\right)} J\left(\sum_{i=1}^{q} \alpha_{i} \tilde{\delta}_{i}+\sum_{i=q+1}^{p} \alpha_{i} \varphi_{i}+v\right) .
$$

Moreover, there exists $c>0$ such that

$$
\|\bar{v}\| \leq c\left(\sum_{i \leq q} \frac{\left|\nabla K\left(a_{i}\right)\right|}{\lambda_{i}}+\frac{1}{\lambda_{i}^{2}}+\sum_{i>q} \frac{1}{\lambda_{i} d_{i}}+\sum_{k \neq r} \varepsilon_{k r}\left(\log \varepsilon_{k r}^{-1}\right)^{1 / 2}\right) .
$$

Here $(\cdot, \cdot)$ denotes the inner scalar product defined on $H^{1}\left(S_{+}^{n}\right)$ by

$$
(u, v)=\int_{S_{+}^{n}} \nabla u \nabla v+\frac{1}{4} n(n-2) \int_{S_{+}^{n}} u v .
$$

Now, we consider the subset of $V(p, \varepsilon)$ given by

$$
V_{b}(p, \varepsilon)=\left\{u \in V(p, \varepsilon): \lambda_{i} d_{i}<\varepsilon \text { for all } i\right\} .
$$

The next propositions are devoted to an useful expansion of $J$ and its gradient near a potential boundary critical point at infinity consisting of $p$ masses. In the sequel, we will write $\tilde{\delta}_{i}$ instead of $\tilde{\delta}_{a_{i}, \lambda_{i}}$.

Proposition 2.4 [Ben Ayed et al. 2002]. For $\varepsilon>0$ small enough and

$$
u=\sum_{i=1}^{p} \alpha_{i} \tilde{\delta}_{a_{i}, \lambda_{i}}+v \in V_{b}(p, \varepsilon)
$$

we have the expansion

$$
J(u)=\frac{\left(S_{n} / 2\right)^{(2 / n)} \sum_{i=1}^{p} \alpha_{i}^{2}}{\left(\sum_{i=1}^{p} \alpha_{i}^{2 n /(n-2)} K\left(a_{i}\right)\right)^{(n-2) / n}}(1+o(1)),
$$

where

$$
S_{n}=\int_{\mathbb{R}^{n}}\left(1+|x|^{2}\right)^{-n} d x .
$$


Proposition 2.5. Let $n=3$ and $u=\sum_{i=1}^{p} \alpha_{i} \tilde{\delta}_{i} \in V_{b}(p, \varepsilon)$. Then

$$
\begin{aligned}
&\left(\nabla J(u), \lambda_{i} \frac{\partial \tilde{\delta}_{i}}{\partial \lambda_{i}}\right)=-c_{1} J(u) \sum_{j \neq i} \alpha_{j} \lambda_{i} \frac{\partial \varepsilon_{i j}}{\partial \lambda_{i}} \\
&+2 J(u)^{4} \alpha_{i}^{5}\left(-\frac{c_{2}}{\lambda_{i}} \frac{\partial K}{\partial v}\left(a_{i}\right)+\frac{c_{3}}{\lambda_{i}^{2}} \Delta K\left(a_{i}\right)\right)+o\left(\sum_{k \neq r} \varepsilon_{k r}+\frac{1}{\lambda_{i}^{2}}\right), \\
&\left(-\nabla J(u), \frac{1}{\lambda_{i}} \frac{\partial \tilde{\delta}_{i}}{\partial a_{i}}\right) \frac{\nabla_{T} K\left(a_{i}\right)}{\left|\nabla_{T} K\left(a_{i}\right)\right|} \geq 2 J(u)^{4} \alpha_{i}^{5}\left(c_{4} \frac{\left|\nabla_{T} K\left(a_{i}\right)\right|}{\lambda_{i}}-c_{5} \frac{\left|D^{2} K\left(a_{i}\right)\right|}{\lambda_{i}^{2}}\right) \\
&+o\left(\frac{1}{\lambda_{i}^{2}}+\sum_{k \neq r} \varepsilon_{k r}\right)+o\left(\sum_{j \neq i} \frac{1}{\lambda_{i}}\left|\frac{\partial \varepsilon_{i j}}{\partial a_{i}}\right|\right),
\end{aligned}
$$

and

$$
\left(\nabla J(u), \tilde{\delta}_{i}\right)=J(u) \alpha_{i} S_{3}\left(1-J(u)^{3} \alpha_{i}^{4} K\left(a_{i}\right)\right)+O\left(\frac{1}{\lambda_{i}}\left|\frac{\partial K}{\partial v}\left(a_{i}\right)\right|+\frac{1}{\lambda_{i}^{2}}+\sum_{j \neq i} \varepsilon_{i j}\right),
$$

where

$$
\begin{array}{ll}
c_{1}=c_{0}^{6} \int_{\mathbb{R}^{3}} \frac{1}{\left(1+|x|^{2}\right)^{5 / 2}} d x=4 \sqrt{3} \pi, & c_{2}=c_{0}^{6} \int_{\mathbb{R}_{+}^{3}} \frac{x_{3}\left(|x|^{2}-1\right)}{\left(1+|x|^{2}\right)^{4}} d x=\frac{\sqrt{3} \pi}{4}, \\
c_{3}=\frac{1}{3} c_{0}^{6} \int_{\mathbb{R}_{+}^{3}} \frac{|x|^{2}\left(|x|^{2}-1\right)}{\left(1+|x|^{2}\right)^{4}} d x, & c_{4}=\frac{1}{6} c_{0}^{6} \int_{\mathbb{R}^{3}} \frac{|x|^{2}}{\left(1+|x|^{2}\right)^{4}} d x, \\
c_{5}=c_{0}^{6} \int_{\mathbb{R}^{3}} \frac{|x|^{2}}{\left(1+|x|^{2}\right)^{3}} d x, & S_{3}=\int_{\mathbb{R}^{3}} \frac{1}{\left(1+|x|^{2}\right)^{3}} d x,
\end{array}
$$

and $c_{0}$ is defined in $(2-1)$.

Proof. The proof is the same as in [Ben Ayed et al. 2002], but here we need to improve some estimates. Observe that

$$
\begin{aligned}
\int_{B\left(a_{i}, 1\right) \cap \mathbb{R}_{+}^{3}}\left(K(x)-K\left(a_{i}\right)\right) \tilde{\delta}_{i}^{5} \lambda_{i} \frac{\partial \tilde{\delta}_{i}}{\partial \lambda_{i}} \\
=\int \nabla K\left(a_{i}\right)\left(x-a_{i}\right) \tilde{\delta}_{i}^{5} \lambda_{i} \frac{\partial \tilde{\delta}_{i}}{\partial \lambda_{i}}+\frac{1}{2} D^{2} K\left(a_{i}\right)\left(x-a_{i}, x-a_{i}\right) \tilde{\delta}_{i}^{5} \lambda_{i} \frac{\partial \tilde{\delta}_{i}}{\partial \lambda_{i}}+o\left(\frac{1}{\lambda_{i}^{2}}\right) \\
=\frac{c_{0}^{6}}{2} \frac{\nabla K\left(a_{i}\right)}{\lambda_{i}} \int_{\mathbb{R}_{+}^{3}} \frac{x\left(1-|x|^{2}\right)}{\left(1+|x|^{2}\right)^{4}}+c_{0}^{6} \frac{\Delta K\left(a_{i}\right)}{12 \lambda_{i}^{2}} \int_{\mathbb{R}_{+}^{3}} \frac{|x|^{2}\left(1-|x|^{2}\right)}{\left(1+|x|^{2}\right)^{4}}+o\left(\frac{1}{\lambda_{i}^{2}}\right) .
\end{aligned}
$$


For $j=1,2$, we denote by $\left(a_{i}\right)_{j}$ the $j$ th component of $a_{i}$.

$$
\begin{aligned}
\int_{B\left(a_{i}, 1\right) \cap \mathbb{R}_{+}^{3}}\left(K(x)-K\left(a_{i}\right)\right) \tilde{\delta}_{i}^{5} & \frac{1}{\lambda_{i}} \frac{\partial \tilde{\delta}_{i}}{\partial\left(a_{i}\right)_{j}}=\int \nabla K\left(a_{i}\right)\left(x-a_{i}\right) \tilde{\delta}_{i}^{5} \frac{1}{\lambda_{i}} \frac{\partial \tilde{\delta}_{i}}{\partial\left(a_{i}\right)_{j}} \\
+ & \frac{1}{2} \int D^{2} K\left(a_{i}\right)\left(x-a_{i}, x-a_{i}\right) \tilde{\delta}_{i}^{5} \frac{1}{\lambda_{i}} \frac{\partial \tilde{\delta}_{i}}{\partial\left(a_{i}\right)_{j}}+o\left(\frac{1}{\lambda_{i}^{2}}\right) .
\end{aligned}
$$

Observe that

$$
\left|\int_{\mathbb{R}_{+}^{3}} D^{2} K\left(a_{i}\right)\left(x-a_{i}, x-a_{i}\right) \tilde{\delta}_{i}^{5} \frac{1}{\lambda_{i}} \frac{\partial \tilde{\delta}_{i}}{\partial\left(a_{i}\right)_{j}}\right| \leq\left|D^{2} K\left(a_{i}\right)\right| \int_{\mathbb{R}_{+}^{3}}\left|x-a_{i}\right|^{2} \tilde{\delta}_{i}^{6}
$$

and

$$
\begin{aligned}
\int_{B\left(a_{i}, 1\right) \cap \mathbb{R}_{+}^{3}} \nabla K\left(a_{i}\right)\left(x-a_{i}\right) \frac{\tilde{\delta}_{i}^{5}}{\lambda_{i}} \frac{\partial \tilde{\delta}_{i}}{\partial\left(a_{i}\right)_{j}} & =\int \nabla K\left(a_{i}\right)\left(x-a_{i}\right) \frac{c_{0}^{6} \lambda_{i}^{4}\left(x-a_{i}\right)_{j}}{\left(1+\lambda_{i}^{2}\left|x-a_{i}\right|^{2}\right)^{4}} \\
& =\frac{\left(\nabla K\left(a_{i}\right)\right)_{j}}{\lambda_{i}} \int_{B\left(0, \lambda_{i}\right) \cap \mathbb{R}_{+}^{3}} \frac{c_{0}^{6} x_{j}^{2}}{\left(1+|x|^{2}\right)^{4}}
\end{aligned}
$$

Following [Ben Ayed et al. 2002], the result is proved.

Let $G$ be the Green's function on $S_{+}^{3}$ and $H$ its regular part defined by

$$
\left\{\begin{aligned}
G(x, y) & =(1-\cos d(x, y))^{-1 / 2}-H(x, y), \\
\Delta H & =0 \text { in } S_{+}^{3}, \quad \frac{\partial G}{\partial v}=0 \text { on } \partial S_{+}^{3} .
\end{aligned}\right.
$$

The following proposition will be as the Proposition 2.5 but the concentration point will be in the interior of $S_{+}^{3}$. We have

Proposition 2.6. Let $n=3$. For $u=\sum_{i \leq q} \alpha_{i} \tilde{\delta}_{i}+\sum_{j=q+1}^{p} \alpha_{j} \varphi_{j} \in V(p, \varepsilon)$, there exist two positive constants $\bar{c}_{1}$ and $\bar{c}_{2}$ such that the following expansion holds:

$$
\begin{aligned}
\left(\nabla J(u), \lambda_{j} \frac{\partial \varphi_{j}}{\partial \lambda_{j}}\right)=J(u)\left(-\bar{c}_{1} \sum_{k \neq j} \alpha_{k} \lambda_{j} \frac{\partial \varepsilon_{j k}}{\partial \lambda_{j}}-\bar{c}_{2} \sum_{k=q+1, k \neq j}^{p} \alpha_{k} \frac{H\left(a_{j}, a_{k}\right)}{\left(\lambda_{j} \lambda_{k}\right)^{1 / 2}}\right) & \\
& +o\left(\sum_{k>q} \frac{1}{\lambda_{k} d_{k}}+\sum_{k \neq r} \varepsilon_{k r}\right) .
\end{aligned}
$$

Now we consider the case where we only have one mass, and we recall the Morse lemma at infinity for $J$, which completely gets rid of the $v$ contribution and shows that the functional behaves, at infinity, as $J\left(\alpha \tilde{\delta}_{\tilde{a}, \tilde{\lambda}}\right)+|V|^{2}$, where $V$ is a variable completely independent of $\tilde{a}$ and $\tilde{\lambda}$. 
Proposition 2.7 [Ben Ayed et al. 2002]. For $\varepsilon>0$ small enough, there is a diffeomorphism

$$
\alpha \tilde{\delta}_{a, \lambda}+v \mapsto\left(\alpha \tilde{\delta}_{\tilde{a}, \tilde{\lambda}}, V\right)
$$

such that

$$
J\left(\alpha \tilde{\delta}_{a, \lambda}+v\right)=J\left(\alpha \tilde{\delta}_{\tilde{a}, \tilde{\lambda}}\right)+|V|^{2}
$$

where $\tilde{a} \in \partial S_{+}^{n}$ and $V$ belongs to a neighborhood of zero in a suitable Hilbert space.

The Morse Lemma above can be improved when the concentration point is near a critical point $y$ of $K_{1}=\left.K\right|_{\partial S_{+}^{n}}$ with $y \in I_{+} \cup I_{0}^{+}$, leading to the following normal form, the proof of which (up to minor modification) is contained in [Ben Ayed et al. 2002].

Proposition 2.8. For $u=\alpha \tilde{\delta}_{\tilde{a}, \tilde{\lambda}} \in V_{b}(1, \varepsilon)$ such that $\tilde{a} \in \mathscr{V}(y, \gamma), y \in I_{+} \underset{\tilde{\tilde{\lambda}}}{\cup} I_{0}^{+}$is a critical point of $K_{1}$, and $\gamma>0$, there is another change of variable $(\tilde{\tilde{a}}, \tilde{\tilde{\lambda}})$ such that, if $y \in I_{+}$,

$$
J(u)=\psi_{1}(\tilde{\tilde{a}}, \tilde{\tilde{\lambda}}):=\frac{\left(S_{n} / 2\right)^{2 / n}}{K(\tilde{\tilde{a}})^{(n-2) / n}}\left(1+(1-\eta) \frac{\bar{c}}{\tilde{\tilde{\lambda}}} \frac{\partial K}{\partial v}(y)\right),
$$

and, if $y \in I_{0}^{+}$,

$$
J(u)=\psi_{2}(\tilde{\tilde{a}}, \tilde{\tilde{\lambda}}):=\frac{\left(S_{n} / 2\right)^{2 / n}}{K(\tilde{\tilde{a}})^{(n-2) / n}}\left(1+\frac{\bar{c}}{\tilde{\tilde{\lambda}}} \frac{\partial K}{\partial v}(\tilde{\tilde{a}})-(1-\eta) \overline{\bar{c}} \frac{\Delta K(\tilde{\tilde{a}})}{\tilde{\tilde{\lambda}}{ }^{2}}\right)
$$

where $\eta$ is a small positive real and $\bar{c}, \overline{\bar{c}}$ are positive constants. Here and in the sequel, $\mathscr{V}(y, \gamma)$ denotes a neighborhood of $y$.

\section{Characterization of the critical points at infinity}

This section is devoted to the characterization of the critical points at infinity of the associated variational problem. We recall that the critical points at infinity of $J$ are the orbits of the gradient flow of $J$ which remain in $V(p, \varepsilon(s))$, where $\varepsilon(s)$ is a given function such that $\varepsilon(s)$ goes to zero when $s$ goes to infinity (see [Bahri 1989]).

First, we are going to construct a global pseudogradient for the functional $J$. Along its flow lines there can be only finitely many isolated blow-up points. Outside $\bigcup_{p} V(p, \varepsilon / 2)$ we use $-\nabla J$, which satisfies the Palais-Smale condition in this set. In $V(p, \varepsilon)$, we use the vector field $W$ constructed below. Such a flow is defined by combining two basic facts. On one hand, the Morse Lemma at infinity moves points and concentrations as follows: points move according to $\nabla_{T} K$, and concentrations move so as to decrease the functional $J$. On the other hand, there is 
another pseudogradient when the $\alpha_{i}$ 's are not in their maximum values. We need to convex-combine both flows to keep the pseudogradient property, to avoid the creation of new asymptotes.

Proposition 3.1. Let $n=3$. For $p \geq 1$, there exist a pseudogradient $W$ and $a$ constant $c>0$ independent of $u=\sum_{i=1}^{q} \alpha_{j_{i}} \tilde{\delta}_{j_{i}}+\sum_{i=q+1}^{p} \alpha_{j_{i}} \varphi_{j_{i}} \in V(p, \varepsilon)$ such that the following properties hold:

$$
\begin{aligned}
& (-\nabla J(u), W) \geq \sum_{i=q+1}^{p} \frac{c}{\lambda_{j_{i}} d_{j_{i}}}+c \sum_{k \neq r} \varepsilon_{k r} \\
& \quad+c \sum_{i=1}^{q}\left(\frac{1}{\lambda_{j_{i}}}\left|\frac{\partial K\left(a_{j_{i}}\right)}{\partial v}\right|+\frac{\left|\nabla_{T} K\left(a_{j_{i}}\right)\right|}{\lambda_{j_{i}}}+\frac{1}{\lambda_{j_{i}}^{2}}+\left|1-J(u)^{3} \alpha_{j_{i}}^{4} K\left(a_{j_{i}}\right)\right|\right) .
\end{aligned}
$$

$$
\begin{aligned}
& \left(-\nabla J(u+\bar{v}), W+\frac{\partial \bar{v}}{\partial\left(\alpha_{i}, a_{i}, \lambda_{i}\right)}(W)\right) \geq \sum_{i=q+1}^{p} \frac{c}{\lambda_{j_{i}} d_{j_{i}}}+c \sum_{k \neq r} \varepsilon_{k r} \\
& \quad+c \sum_{i=1}^{q}\left(\frac{1}{\lambda_{j_{i}}}\left|\frac{\partial K\left(a_{j_{i}}\right)}{\partial v}\right|+\frac{\left|\nabla_{T} K\left(a_{j_{i}}\right)\right|}{\lambda_{j_{i}}}+\frac{1}{\lambda_{j_{i}}^{2}}+\left|1-J(u)^{3} \alpha_{j_{i}}^{4} K\left(a_{j_{i}}\right)\right|\right) .
\end{aligned}
$$

(iii) $|W|$ is bounded.

Furthermore, the only cases where the maximum of the $\lambda_{i}$ is not bounded along the flow lines of $W$ are when:

- $p=1$ and the concentration point a is near a critical point $y$ of $K_{1}$ with $(\partial K / \partial v)(y)=0$ and $-\Delta K(y)>0$.

- $p \geq 1$ and each point $a_{i}$ is close to a critical point $y_{j_{i}}$ of $K_{1}$ with $j_{i} \neq j_{k}$ for $i \neq k$, and the smallest eigenvalue $\rho\left(y_{i_{1}}, \ldots, y_{i_{p}}\right)$ of $M\left(y_{i_{1}}, \ldots, y_{i_{p}}\right)$ is positive.

Before giving the proof, we state a corollary of Proposition 3.1.

Corollary 3.2. Let $n=3$. Assume that $J$ has no critical point in $\Sigma^{+}$. Then, the only critical points at infinity of $J$ correspond to:

- $\tilde{\delta}_{(y, \infty)}$, where $y$ is a critical point of $K_{1}$ satisfying $(\partial K / \partial \nu)(y)=0$ and $-\Delta K(y)>0$.

- $\sum_{i=1}^{p} K(y)^{-1 / 4} \tilde{\delta}_{\left(y_{j_{i}}, \infty\right)}$, where $p \geq 1$ and the $y_{j_{i}}$ satisfy $(\partial K / \partial v)\left(y_{j_{i}}\right)>0$ and $\rho\left(y_{j_{1}}, \ldots, y_{j_{p}}\right)>0$.

Proof of Proposition 3.1. Without loss of generality, we assume that $\lambda_{1} \leq \cdots \leq \lambda_{p}$. For $M$ and $C_{1}$ large positive constants, and $\eta$ a small positive constant such that 
$\eta M$ is large, we define

$$
\begin{aligned}
D & =\{1\} \cup\left\{i: \lambda_{k} \leq M \lambda_{k-1} \text { for all } 2 \leq k \leq i\right\}, \\
E & =\left\{i: d\left(a_{i}, y\right)>\frac{1}{2} \eta \text { for all } y \text { such that } \nabla_{T} K(y)=0\right\}, \\
F_{k} & =\left\{i: d\left(a_{i}, y_{k}\right)<\eta\right\}, \text { where } y_{k} \text { is a critical point of } K_{1}, \\
L & =\left\{i: d_{i}=0,\left|1-J(u)^{3} \alpha_{i}^{4} K\left(a_{i}\right)\right| \geq \frac{C_{1}}{\lambda_{i}}\left(\left|\frac{\partial K\left(a_{i}\right)}{\partial v}\right|+\left|\nabla_{T} K\left(a_{i}\right)\right|+\frac{1}{\lambda_{i}}\right)\right\} .
\end{aligned}
$$

For $u \in V_{b}(p, \varepsilon)$, seven cases may occur. We will define a vector field in each case and $W$ will be a convex combination of all cases. In the following, we set

$$
\begin{aligned}
& N_{0}=\left\{i \text { : there is } y \text { such that } \nabla_{T} K(y)=0,(\partial K / \partial v)(y)=0 \text { and } d\left(a_{i}, y\right)<\eta\right\}, \\
& N_{-}=\left\{i \text { : there is } y \text { such that } \nabla_{T} K(y)=0,(\partial K / \partial v)(y)<0 \text { and } d\left(a_{i}, y\right)<\eta\right\}, \\
& N_{+}=\left\{i \text { : there is } y \text { such that } \nabla_{T} K(y)=0,(\partial K / \partial v)(y)>0 \text { and } d\left(a_{i}, y\right)<\eta\right\} .
\end{aligned}
$$

Case 1. $u \in V_{b}(p, \varepsilon)$ and there exist $i, j \in D$ such that $d\left(a_{i}, a_{j}\right)<4 \eta$.

Let $i_{0}$ be the least index in $D$ such that there exists $j_{0}<i_{0}$ satisfying $d\left(a_{i_{0}}, a_{j_{0}}\right)<4 \eta$ (it follows that $\lambda_{j_{0}} \leq \lambda_{i_{0}}$ and, for any $i, j<i_{0}$, we have $d\left(a_{i}, a_{j}\right) \geq 4 \eta$ ). In this case, we define

$$
Z_{1}=-\sum_{k=i_{0}}^{p} 2^{k} \alpha_{k} \lambda_{k} \frac{\partial \tilde{\delta}_{k}}{\partial \lambda_{k}}, \quad Z_{\alpha}=-\sum_{i \in L} \operatorname{sign}\left(1-J(u)^{3} \alpha_{i}^{4} K\left(a_{i}\right)\right) \tilde{\delta}_{i} .
$$

Using Proposition 2.5, we derive that

$\left(-\nabla J(u), Z_{\alpha}\right) \geq c \sum_{i \in L}\left|1-J(u)^{3} \alpha_{i}^{4} K\left(a_{i}\right)\right|+\sum_{i \in L} O\left(\frac{1}{\lambda_{i}}\left|\frac{\partial K\left(a_{i}\right)}{\partial v}\right|+\frac{1}{\lambda_{i}^{2}}+\sum_{k \neq i} \varepsilon_{k i}\right)$.

Furthermore, an easy computation shows that

(3-1) $\quad-\lambda_{i} \frac{\partial \varepsilon_{i j}}{\partial \lambda_{i}}=\frac{1}{2} \varepsilon_{i j}\left(1-2 \frac{\lambda_{j}}{\lambda_{i}} \varepsilon_{i j}^{2}\right)$ and $\sum_{k=i, j}-2^{k} \lambda_{k} \frac{\partial \varepsilon_{i j}}{\partial \lambda_{k}} \geq \varepsilon_{i j}(1+o(1))$.

Thus, also using Proposition 2.5, we obtain

$$
\left(-\nabla J(u), Z_{1}\right) \geq c \sum_{k \geq i_{0}}\left(\sum_{r \neq k} \varepsilon_{k r}+O\left(\frac{1}{\lambda_{k}}\right)\right)+o\left(\sum_{r \neq l} \varepsilon_{r l}\right) .
$$

Observe that $1 / \lambda_{i_{0}}=o\left(\varepsilon_{i_{0} j_{0}}\right)$; indeed, since $\lambda_{j_{0}} \leq \lambda_{i_{0}}$,

$$
\frac{1}{\lambda_{i_{0}}^{2} \varepsilon_{i_{0} j_{0}}^{2}}=\frac{1}{\lambda_{i_{0}} \lambda_{j_{0}}}+\frac{\lambda_{j_{0}}}{\lambda_{i_{0}}^{3}}+\frac{\lambda_{j_{0}}}{2 \lambda_{i_{0}}}\left(1-\cos d\left(a_{i_{0}}, a_{j_{0}}\right)\right) \leq o(1)+4 \eta^{2}=o(1)
$$


Thus, since for $k \geq i_{0}$ we have $\lambda_{k} \geq \lambda_{i_{0}}$, (3-2) becomes

$$
\left(-\nabla J(u), Z_{1}\right) \geq c \sum_{k \geq i_{0}, r \neq k} \varepsilon_{k r}+\frac{c}{\lambda_{i_{0}}}+o\left(\sum_{l \neq r} \varepsilon_{l r}\right) .
$$

Since $i_{0} \in D$, we can make $1 / \lambda_{1}$ appear in the lower bound of (3-4), and hence also all the other $1 / \lambda_{i}$. Furthermore, for $i, j<i_{0}$, we have $d\left(a_{i}, a_{j}\right) \geq 4 \eta$, then from $1 / \lambda_{i}$ and $1 / \lambda_{j}$ we can make $\varepsilon_{i j}$ appear in the lower bound. Thus, we derive

$$
\left(-\nabla J(u), Z_{1}\right) \geq \sum_{i=1}^{p} \frac{c}{\lambda_{i}}\left(\left|\frac{\partial K\left(a_{i}\right)}{\partial v}\right|+\left|\nabla_{T} K\left(a_{i}\right)\right|+\frac{1}{\lambda_{i}}\right)+c \sum_{k \neq r} \varepsilon_{k r} .
$$

Now, by defining $W_{1}=M_{1} Z_{1}+Z_{\alpha}$, for some large positive constant $M_{1}$, we obtain the desired inequality in (i) by replacing $W$ with $W_{1}$.

Case 2. $u \in V_{b}(p, \varepsilon), d\left(a_{i}, a_{j}\right) \geq 4 \eta$ for each $i, j \in D$, and $D \cap E \neq \varnothing$. Define

$$
Z_{2}=\sum_{i \in D \cap E} \frac{1}{\lambda_{i}} \frac{\partial \tilde{\delta}_{i}}{\partial a_{i}} \frac{\nabla_{T} K\left(a_{i}\right)}{\left|\nabla_{T} K\left(a_{i}\right)\right|}-\gamma \sum_{i \notin D} 2^{i} \alpha_{i} \lambda_{i} \frac{\partial \tilde{\delta}_{i}}{\partial \lambda_{i}},
$$

where $\gamma$ is a large positive constant. Using Proposition 2.5, (3-1) and the fact that $\left|\nabla_{T} K\left(a_{i}\right)\right| \geq c \eta$ for $i \in E$, we obtain

$$
\begin{aligned}
\left(-\nabla J(u), Z_{2}\right) \geq c \sum_{i \in D \cap E}( & \left.\frac{c \eta}{\lambda_{i}}+O\left(\sum_{j \in D} \frac{1}{\lambda_{i}}\left|\frac{\partial \varepsilon_{i j}}{\partial a_{i}}\right|+\sum_{j \notin D} \varepsilon_{i j}\right)\right) \\
& +\gamma c \sum_{i \notin D, j \neq i} \varepsilon_{i j}+O\left(\sum_{k \notin D} \frac{\gamma}{\lambda_{k}}\right)+o\left(\sum_{l \neq r} \varepsilon_{l r}\right) .
\end{aligned}
$$

Observe that, for $k \notin D$ and $i \in D$, if we choose $\gamma$ so that $\gamma /(\eta M)$ is small, we derive that $\gamma / \lambda_{k} \leq \gamma /\left(M \lambda_{i}\right)=o\left(\eta / \lambda_{i}\right)$. Furthermore, for $i, j \in D$, since $d\left(a_{i}, a_{j}\right) \geq 4 \eta$, we see that

$$
\frac{1}{\lambda_{i}}\left|\frac{\partial \varepsilon_{i j}}{\partial a_{i}}\right| \leq c \lambda_{j} d\left(a_{i}, a_{j}\right) \varepsilon_{i j}^{3} \leq \frac{c}{\lambda_{i}^{3 / 2} \lambda_{j}^{1 / 2} \eta^{2}}=o\left(\frac{\eta}{\lambda_{i}}\right) .
$$

Thus (3-6) becomes

$$
\left(-\nabla J(u), Z_{2}\right) \geq \sum_{i \in D \cap E} \frac{c}{\lambda_{i}}+c \sum_{i \notin D, j \neq i} \varepsilon_{i j}+o\left(\sum_{l \neq r} \varepsilon_{l r}\right) .
$$

As in Case 1, we obtain (3-5) with $Z_{2}$ instead of $Z_{1}$ and define $W_{2}=M_{1} Z_{2}+Z_{\alpha}$, thus deriving the desired inequality in (i) by replacing $W$ with $W_{2}$. 
Case 3. $u \in V_{b}(p, \varepsilon)$, for each $i, j \in D$ we have $d\left(a_{i}, a_{j}\right) \geq 4 \eta$, and $D \cap N_{-} \neq \varnothing$. Define

$$
Z_{3}=-\sum_{i \in D \cap N_{-}} \lambda_{i} \frac{\partial \tilde{\delta}_{i}}{\partial \lambda_{i}}-\sum_{i \notin D} 2^{i} \alpha_{i} \lambda_{i} \frac{\partial \tilde{\delta}_{i}}{\partial \lambda_{i}} .
$$

Using Proposition 2.5 and (3-1), we derive

$$
\left(-\nabla J(u), Z_{3}\right) \geq \sum_{i \in D \cap N_{-}} c\left(\frac{1}{\lambda_{i}}+\sum_{j \in D} \varepsilon_{i j}\right)+c \sum_{k \notin D, j \neq k} \varepsilon_{k j}+O\left(\sum_{k \notin D} \frac{1}{\lambda_{k}}\right)+o\left(\sum_{l \neq r} \varepsilon_{l r}\right) .
$$

As in Case 1, we obtain (3-5) with $Z_{3}$ instead of $Z_{1}$, and define $W_{3}=M_{1} Z_{3}+Z_{\alpha}$, thus deriving the desired inequality in (i) by replacing $W$ with $W_{3}$.

Case 4. $u \in V_{b}(p, \varepsilon), D \cap E=D \cap N_{-}=\varnothing \neq D \cap N_{0},\{1\} \varsubsetneqq D$, and $d\left(a_{i}, a_{j}\right) \geq 4 \eta$ for each $i, j \in D$. Then $D \subset \bigcup_{k} F_{k}$, since $D \cap E=\varnothing$. Furthermore, since $d\left(a_{i}, a_{j}\right) \geq 4 \eta$ for each $i, j \in D$, we conclude that $d\left(a_{i}, a_{j}\right) \geq \frac{1}{2} \min \left\{d\left(y_{k}, y_{r}\right): k \neq r\right\}$. In this case, we define

$$
Z_{4}=-\sum_{i \in D \cap N_{0}} \lambda_{i} \frac{\partial \tilde{\delta}_{i}}{\partial \lambda_{i}}-\sum_{i \notin D} 2^{i} \alpha_{i} \lambda_{i} \frac{\partial \tilde{\delta}_{i}}{\partial \lambda_{i}} .
$$

Using Proposition 2.5 and (3-1), we derive

$$
\begin{aligned}
\left(-\nabla J(u), Z_{4}\right) \geq c \sum_{i \in D \cap N_{0}}\left(\sum_{j \in D} \varepsilon_{i j}\right. & \left.+O\left(\frac{1}{\lambda_{i}}\left|\frac{\partial K}{\partial v}\left(a_{i}\right)\right|+\frac{1}{\lambda_{i}^{2}}\right)\right) \\
& +c \sum_{k \notin D, j \neq k}\left(\varepsilon_{k j}+O\left(\frac{1}{\lambda_{k}}\right)\right)+o\left(\sum_{l \neq r} \varepsilon_{l r}\right) .
\end{aligned}
$$

Since $D \neq\{1\}$, for any $i \in D \cap N_{0}$, either $i-1$ or $i+1$ belongs to $D$. As in (3-3), it is easy to see that $1 / \lambda_{i} \leq c \sqrt{M} \varepsilon_{i j}$ where $j=i-1$ if $i-1 \in D$, if not $j=i+1$. Thus, choosing $\eta$ small enough, we derive that $\left|(\partial K / \partial v)\left(a_{i}\right)\right|$ is small with respect to $1 /(c \sqrt{M})$ if $\eta^{2} M$ is small. Furthermore, for $k \notin D$ and $i \in D \cap N_{0}$, we have $1 / \lambda_{k} \leq 1 /\left(M \lambda_{i}\right)=o\left(\varepsilon_{i j}\right)$. Therefore, (3-7) becomes

$$
\left(-\nabla J(u), Z_{4}\right) \geq c \sum_{i \in D \cap N_{0}}\left(\frac{1}{\lambda_{i}}+\sum_{j \in D} \varepsilon_{i j}\right)+c \sum_{k \notin D, j \neq k} \varepsilon_{k j}+o\left(\sum_{l \neq r} \varepsilon_{l r}\right) .
$$

We conclude as in the second and the third cases, defining $W_{4}=M_{1} Z_{4}+Z_{\alpha}$.

Case 5. $u \in V_{b}(p, \varepsilon), d\left(a_{i}, a_{j}\right) \geq 4 \eta$ for each $i, j \in D, D \cap E=D \cap N_{-}=\varnothing$ and $D=\{1\} \subset N_{0}$. Then $a_{1}$ is near a critical point $y_{i_{1}}$ of $K_{1}$ satisfying $\left(A_{2}\right)$. Define

$$
Z_{5}=\operatorname{sign}\left(-\Delta K\left(y_{i_{1}}\right)\right) \lambda_{1} \frac{\partial \tilde{\delta}_{1}}{\partial \lambda_{1}}+\frac{\bar{c}_{4}}{\lambda_{1}} \frac{\partial \tilde{\delta}_{1}}{\partial a_{1}} \frac{\nabla_{T} K\left(a_{1}\right)}{\left|\nabla_{T} K\left(a_{1}\right)\right|}-\sum_{i \geq 2} 2^{i} \alpha_{i} \lambda_{i} \frac{\partial \tilde{\delta}_{i}}{\partial \lambda_{i}}
$$


where $\bar{c}_{4}$ is a small positive constant. Using Proposition 2.5, (3-1) and assumption $\left(A_{2}\right)$, we derive that

$$
\left(-\nabla J(u), Z_{5}\right) \geq \frac{c}{\lambda_{1}}\left|\frac{\partial K}{\partial v}\left(a_{1}\right)\right|+\frac{c}{\lambda_{1}^{2}}+c \frac{\left|\nabla_{T} K\left(a_{1}\right)\right|}{\lambda_{1}}+c \sum_{k \neq r} \varepsilon_{k r}+O\left(\frac{1}{\lambda_{2}}\right) .
$$

As in (3-3), since $2 \notin D$, it is easy to see that $1 / \lambda_{2}=o\left(\varepsilon_{12}\right)$. Thus, we can make $1 / \lambda_{2}$ appear in the lower bound. Now, define $W_{5}=M_{1} Z_{5}+Z_{\alpha}$ and we conclude as in the preceding cases.

Case 6. $u \in V_{b}(p, \varepsilon)$, for each $i, j \in D$, we have $d\left(a_{i}, a_{j}\right) \geq 4 \eta$ and $D \subset N_{+}$.

In this case, for each $k, B\left(y_{k}, \eta\right)$ contains at most one of the $a_{i}$. Let us denote by $y_{i_{1}}, \ldots, y_{i_{q}}$ the critical points such that $a_{j} \in B\left(y_{i_{j}}, \eta\right)$, where $q=$ card $D$. The vector field will depend on the sign of the smallest eigenvalue $\rho$ of the matrix $M\left(y_{i_{1}}, \ldots, y_{i_{q}}\right)$, defined in (1-5). Two subcases may occur.

Case 6(a). If $\rho>0$, we define

$$
Z_{6}^{1}=\sum_{i \in D} \alpha_{i} \lambda_{i} \frac{\partial \tilde{\delta}_{i}}{\partial \lambda_{i}}-\sum_{i \notin D} 2^{i} \alpha_{i} \lambda_{i} \frac{\partial \tilde{\delta}_{i}}{\partial \lambda_{i}}
$$

For $i, k \in D$, we have $d\left(a_{i}, a_{k}\right)=d\left(y_{j_{i}}, y_{j_{k}}\right)+o(1)$. Thus, (3-1) implies (3-8)

$$
-\lambda_{i} \frac{\partial \varepsilon_{i k}}{\partial \lambda_{i}}=\frac{\sqrt{2}}{2 \sqrt{\lambda_{i} \lambda_{k}\left(1-\cos d\left(y_{j_{i}}, y_{j_{k}}\right)\right)}}+o\left(\varepsilon_{i k}\right)=\frac{\sqrt{2} G_{1}\left(y_{j_{i}}, y_{j_{k}}\right)}{2 \sqrt{\lambda_{i} \lambda_{k}}}+o\left(\varepsilon_{i k}\right) .
$$

Using Proposition 2.5, (3-1), (3-8) and the fact that $J(u)^{3} \alpha_{i}^{4} K\left(a_{i}\right)=1+o(1)$, we obtain

$$
\begin{aligned}
& \left(-\nabla J(u), Z_{6}^{1}\right) \\
& \begin{array}{r}
\geq \frac{\pi \sqrt{3}}{2 \sqrt{J(u)}} \sum_{i \in D}\left(\frac{(\partial K / \partial v)\left(y_{j_{i}}\right)}{\lambda_{i} K\left(y_{j_{i}}\right)^{3 / 2}}-\sum_{k \in D, k \neq i} \frac{4 \sqrt{2} G_{1}\left(y_{j_{i}}, y_{j_{k}}\right)}{\left(K\left(y_{j_{i}}\right) K\left(y_{j_{k}}\right)\right)^{1 / 4} \sqrt{\lambda_{i} \lambda_{k}}}\right) \\
\quad+o\left(\sum_{i, k \in D} \varepsilon_{i k}+\sum_{i \in D} \frac{1}{\lambda_{i}}\right)+c \sum_{k \notin D, k \neq r} \varepsilon_{k r}+O\left(\sum_{k \notin D} \frac{1}{\lambda_{k}}\right) \\
\geq \frac{\pi \sqrt{3}}{2 \sqrt{J(u)}} \Lambda^{t} M\left(y_{j_{1}}, \ldots, y_{j_{q}}\right) \Lambda+o\left(\sum_{i, k \in D} \varepsilon_{i k}+\sum_{i \in D} \frac{1}{\lambda_{i}}\right) \\
+c \sum_{k \notin D, k \neq r} \varepsilon_{k r}+O\left(\sum_{k \notin D} \frac{1}{\lambda_{k}}\right),
\end{array}
\end{aligned}
$$


where $\Lambda=\left(\lambda_{i_{1}}^{-1 / 2}, \ldots, \lambda_{i_{q}}^{-1 / 2}\right)^{t}$. Since $\rho>0$,

$$
\left(-\nabla J(u), Z_{6}^{1}\right) \geq \sum_{i \in D} \frac{c}{\lambda_{i}}+c \sum_{k \notin D, k \neq r} \varepsilon_{k r}+O\left(\sum_{k \notin D} \frac{1}{\lambda_{k}}\right) .
$$

We define $W_{6}^{1}=M_{1} Z_{6}^{1}+Z_{\alpha}$ and proceed as in the preceding cases.

Case 6(b). If $\rho<0$, denote by $e$ the eigenvector associated to $\rho$, chosen so that $\|e\|=1$ and all of its components are positive. Now, as in [Ben Ayed et al. 1996], we introduce a small neighborhood of $e$ as follows. For $\gamma^{\prime}>0$ small,

$$
\begin{aligned}
B\left(e, \gamma^{\prime}\right) & =\left\{x \in S^{q-1}:\|x-e\|<\gamma^{\prime}\right\}, \\
T_{1}\left(e, \gamma^{\prime}\right) & =\left\{x \in \mathbb{R}^{q}-\{0\}:\|x\|^{-1} x \in B\left(e, \gamma^{\prime}\right)\right\} .
\end{aligned}
$$

We will choose $\gamma^{\prime}$ so that $x^{t} M x<\rho / 2$ for each $x \in B\left(e, \gamma^{\prime}\right)$. There are two cases to consider:

- If $\Lambda \in T_{1}\left(e, \gamma^{\prime}\right)$, we decrease the $\lambda_{i}$ by defining

$$
Y=-\sum_{i \in D} \alpha_{i} \lambda_{i} \frac{\partial \tilde{\delta}_{i}}{\partial \lambda_{i}}
$$

- Otherwise, we define $Y$ by moving the vector $\Lambda$ to the vector $e$ on the sphere of radius $\|\Lambda\|$ (see the vector field $X_{3}$ in [Ben Ayed et al. 1996]).

Now, define $Z_{6}^{2}=Y-\sqrt{M} \sum_{i \notin D} 2^{i} \alpha_{i} \lambda_{i} \partial \tilde{\delta}_{i} / \partial \lambda_{i}$. By [Ben Ayed et al. 1996] and Proposition 2.5, we obtain

$$
\begin{aligned}
\left(-\nabla J(u), Z_{6}^{2}\right) & \geq c \sum_{i \in D}\left(\frac{1}{\lambda_{i}}+O\left(\sum_{k \notin D} \varepsilon_{k i}\right)\right)+\sqrt{M} c \sum_{i \notin D}\left(\sum_{j \neq i} \varepsilon_{i j}+O\left(\frac{1}{\lambda_{i}}\right)\right) \\
& \geq c \sum_{i=1}^{p} \frac{1}{\lambda_{i}}+c \sum_{k \neq r} \varepsilon_{k r} .
\end{aligned}
$$

We conclude as in the preceding cases by defining $W_{6}^{2}=M_{1} Z_{6}^{2}+Z_{\alpha}$.

Case 7. $u \in V(p, \varepsilon)-V_{b}(p, \varepsilon)$. Let $i_{1}, \ldots, i_{q}$ be such that $d_{i_{j}}=0$ and $i_{q+1}, \ldots, i_{p}$ be such that $\lambda_{i_{j}} d_{i_{j}}>\varepsilon^{-1}$. We denote by $u_{1}$ the function $\sum_{k=1}^{q} \alpha_{i_{k}} \tilde{\delta}_{i_{k}}$, which belongs to $V_{b}(q, \varepsilon)$, and define the vector field

$$
Z_{7}^{1}=-\sum_{k=q+1}^{p} 2^{k} \alpha_{i_{k}} \lambda_{i_{k}} \frac{\partial \varphi_{i_{k}}}{\partial \lambda_{i_{k}}}
$$


Using Proposition 2.6, we derive that

$(3-11) \quad\left(-\nabla J(u), Z_{7}^{1}\right)$

$$
\begin{aligned}
=J(u) \sum_{k>q} \alpha_{i_{k}} 2^{k}\left(-\bar{c}_{1} \sum_{j \neq i_{k}} \alpha_{j} \lambda_{i_{k}} \frac{\partial \varepsilon_{j i_{k}}}{\partial \lambda_{i_{k}}}\right. & \left.-\bar{c}_{2} \sum_{j>q} \alpha_{j} \frac{H\left(a_{j}, a_{i_{k}}\right)}{\sqrt{\lambda_{j} \lambda_{i_{k}}}}\right) \\
& +o\left(\sum_{l \neq r} \varepsilon_{l r}+\sum_{k>q} \frac{1}{\lambda_{i_{k}} d_{i_{k}}}\right) .
\end{aligned}
$$

Using the fact that $H(a, \cdot)<-c / d_{a}$ and $\varepsilon_{j i_{k}}^{2} \lambda_{j} / \lambda_{i_{k}}=o(1)$ for $j \in\left\{i_{1}, \ldots, i_{q}\right\}$, (3-1) and (3-11) imply that

$$
\left(-\nabla J(u), Z_{7}^{1}\right) \geq c \sum_{k=q+1}^{p}\left(\frac{1}{\lambda_{i_{k}} d_{i_{k}}}+\sum_{j \neq i_{k}} \varepsilon_{j i_{k}}\right)+o\left(\sum_{l \neq r} \varepsilon_{l r}\right) .
$$

There are two subcases to consider.

Case 7(a). $D \cap\left\{i_{q+1}, \ldots, i_{p}\right\}=\varnothing$. Here we use the previous construction of $u_{1} \in V_{b}(q, \varepsilon)$, that is, we apply the construction only to the indices $i_{1}, \ldots, i_{q}$, forgetting the other indices. Let $Z_{7}^{*}\left(u_{1}\right)$ be the vector field thus defined. From the previous estimates, we have

$\left(-\nabla J(u), Z_{7}^{*}\right)$

$$
\begin{aligned}
\geq c \sum_{i=1}^{q}\left(\frac{1}{\lambda_{j_{i}}}\left|\frac{\partial K\left(a_{j_{i}}\right)}{\partial v}\right|+\frac{\left|\nabla_{T} K\left(a_{j_{i}}\right)\right|}{\lambda_{j_{i}}}+\frac{1}{\lambda_{j_{i}}^{2}}\right. & \left.+\left|1-J(u)^{3} \alpha_{j_{i}}^{4} K\left(a_{j_{i}}\right)\right|\right) \\
& +c \sum_{k \neq r \leq q} \varepsilon_{k r}+O\left(\sum_{k \leq q, r>q} \varepsilon_{k r}\right) .
\end{aligned}
$$

Thus, by setting $W_{7}^{1}=M_{2} Z_{7}^{1}+Z_{7}^{*}\left(u_{1}\right)$ and choosing $M_{2}$ large enough, we derive the desired estimate in claim (i) of Proposition 3.1.

Case 7(b). $D \cap\left\{i_{q+1}, \ldots, i_{p}\right\} \neq \varnothing$. Here we can make the $1 / \lambda_{i_{k}}$, for $k \leq q$, appear in the lower bound of (3-12). Furthermore, define

$$
Z_{7}^{2}=-\sum_{i=1}^{p} 2^{i} \alpha_{i} \lambda_{i} \frac{\partial \varphi_{i}}{\partial \lambda_{i}}
$$

(observe that $\varphi_{i}=\tilde{\delta}_{i}$ if $a_{i} \in \partial S_{+}^{3}$ ). Using Propositions 2.5 and 2.6, we derive that

$$
\left(-\nabla J(u), Z_{7}^{2}\right) \geq c \sum_{k \neq r} \varepsilon_{k r}+c \sum_{k>q} \frac{c}{\lambda_{i_{k}} d_{i_{k}}}+O\left(\sum_{k \leq q} \frac{1}{\lambda_{i_{k}}}\right) .
$$

Thus, by setting $W_{7}^{2}=M_{2} Z_{7}^{1}+Z_{7}^{2}$, we get claim (i) of Proposition 3.1. 
The vector field $W$ will be a convex combination of the above vector fields $W_{1}, \ldots, W_{5}, W_{6}^{i}, W_{7}^{i}$, and will claim (i). Claim (ii) relies on the estimates of $\|\nabla J(u+\bar{v})\|^{2},\|\bar{v}\|^{2}$ and $J^{\prime \prime}(u) \bar{v} W$, which are very small with respect to the lower bound of (i) (see [Ben Ayed et al. 1996, Lemma B.4]). Hence claim (ii) follows, and it only remains to prove claim (iii). Observe that, in cases 1, 2, 3, 4, and 7, the maximum of the $\lambda_{i}$ is a decreasing function along the flow lines. In Case 5, the maximum of the $\lambda_{i}$ increases only if $p=1$ and $a_{1}$ is near a critical point $y$ of $K_{1}$ with $(\partial K / \partial v)(y)=0$ and $-\Delta K(y)>0$. But in Case 6 , the maximum of the $\lambda_{i}$ increases only if $D^{c}=\varnothing$ and $\rho>0$. The proof of Proposition 3.1 is thereby completed.

\section{Proofs of the Theorems}

Proof of Theorem 1.1. For $\eta_{1}>0$ small enough, we introduce the following neighborhood of $\Sigma^{+}$:

$$
V_{\eta_{1}}\left(\Sigma^{+}\right)=\left\{u \in \Sigma: e^{J(u)} J(u)^{2}\left|u^{-}\right|_{L^{6}}^{2}<\eta_{1}\right\},
$$

where $u^{-}=\max (0,-u)$.

Recall that in Proposition 3.1 we constructed a vector field $W$ defined in $V(p, \varepsilon)$ for $p \geq 1$. Outside $\bigcup_{p \geq 1} V(p, \varepsilon / 2)$, we will use $-\nabla J$ and our global vector field $Z$ will be built using a convex combination of $W$ and $-\nabla J$. Observe that $V_{\eta_{1}}\left(\Sigma^{+}\right)$is invariant under the flow line generated by $Z$ (we can repeat the proof of [Ben Ayed et al. 1996, Lemma 4.1] in this case). Since $V_{\eta_{1}}\left(\Sigma^{+}\right)$is contractible, we have $\chi\left(V_{\eta_{1}}\left(\Sigma^{+}\right)\right)=1$, where $\chi$ is the Euler-Poincare characteristic.

We will compute this number using the level sets of $J$. Arguing by contradiction, we suppose that $J$ has no critical points in $V_{\eta_{1}}\left(\Sigma^{+}\right)$. It follows from Corollary 3.2 that the only critical points at infinity of $J$ in $V_{\eta_{1}}\left(\Sigma^{+}\right)$correspond to:

- $\tilde{\delta}_{(y, \infty)}$, where $y$ is a critical point of $K_{1}$ such that $(\partial K / \partial v)(y)=0$ and $-\Delta K(y)>0$. Such a critical point at infinity has a Morse index equal to $2-i(y)$, where $i(y)$ denotes the Morse index of $K_{1}$ at $y$.

- $\sum_{j=1}^{p} K\left(y_{i_{j}}\right)^{-1 / 4} \tilde{\delta}_{\left(y_{i_{j}}, \infty\right)}$, with $p \in \mathbb{N}^{*}$ and $\rho\left(y_{i_{1}}, \ldots, y_{i_{p}}\right)>0$. Such a critical point at infinity has a Morse index equal to $3 p-1-\sum_{j=1}^{p} i\left(y_{i_{j}}\right)$.

Using the vector field $Z$, we have that $V_{\eta_{1}}\left(\Sigma^{+}\right)$retracts by deformation onto $\bigcup W_{u}\left(w_{\infty}\right)$ (see [Bahri and Rabinowitz 1991, Sections 7 and 8]), where $W_{u}\left(w_{\infty}\right)$ is the unstable manifold at infinity of the critical point at infinity $w_{\infty}$. Then

$$
1=\chi\left(V_{\eta_{1}}\left(\Sigma^{+}\right)\right)=\sum_{y \in I_{0}^{+}}(-1)^{2-i(y)}+\sum_{p=1}^{\operatorname{card} I_{+}} \sum_{\substack{\tau_{p}=\left(i_{1}, \ldots, i_{p}\right) \in \mathscr{F}_{p} \\ \rho\left(\tau_{p}\right)>0}}(-1)^{3 p-1-\sum_{j=1}^{p} i\left(y_{i_{j}}\right)},
$$


which contradicts the assumption of our theorem. Thus there exists a critical point of $J$ in $V_{\eta_{1}}\left(\Sigma^{+}\right)$. Arguing as in [Ben Ayed et al. 1996], we prove that this critical point is a positive function and hence our result follows.

Proof of Corollary 1.2. Using the assumption $\left(A_{3}\right)$, we derive that $\mathscr{F}_{p}$ is empty for each $p$, and the result follows from Theorem 1.1.

Proof of Theorem 1.3. We argue by contradiction. Suppose that (1-4) has no solution. Let $c_{1}=(3 / 2)\left(S_{3} / 2\right)^{2 / 3}$. Using the expansion of $J$ (see Proposition 2.4), it follows that there exists a constant $c_{0}$ independent of $K$ such that if

$$
\left|K_{1}-1\right|_{L^{\infty}\left(\partial S_{+}^{3}\right)} \leq c_{0},
$$

then $J(u)<c_{1}$ for each $u \in V_{b}(1, \varepsilon)$, and $J(u)>c_{1}$ for each $u \in V_{b}(p, \varepsilon)$ with $p \geq 2$, where $\varepsilon$ is a small positive constant. Then it follows from Corollary 3.2 that the critical points at infinity of $J$ under the level $c_{1}$ are in one to one correspondence with the critical points $y_{i}$ of $K_{1}$ such that $y_{i} \in I_{+} \cup I_{0}^{+}$. The unstable manifold at infinity of such critical points at infinity, $W_{u}\left(y_{i}\right)_{\infty}$, for $y_{i} \in I_{+} \cup I_{0}^{+}$, can be described using Proposition 2.8 as the product of $W_{s}\left(y_{i}\right)$ (for a pseudogradient of $K_{1}$ ) with $[A,+\infty$, the domain of the variable $\lambda$, for some suitably large positive number $A$.

Since $J$ has no critical points in $\Sigma^{+}$, it follows that $J_{c_{1}}=\left\{u \in \Sigma^{+}: J(u) \leq c_{1}\right\}$ retracts by deformation onto $X_{\infty}=\bigcup_{y_{i} \in I_{+} \cup I_{0}^{+}} W_{u}\left(y_{i}\right)_{\infty}$ (see [Bahri and Rabinowitz 1991, Sections 7 and 8]) which can be parametrized by $X_{1} \times[A,+\infty$, as noted.

At the same time, $X_{\infty}$ is contractible in $J_{c_{1}}$, for the following reason. From $\left(A_{4}\right)$, we deduce that $X_{1} \neq \partial S_{+}^{3} \equiv S^{2}$, then $X_{1}$ is contractible in $S^{2}$. It follows that there exists a continuous contraction $h:[0,1] \times X \rightarrow S^{2}$ such that, for any $a \in X_{1}, h(0, a)=a$ and $h(1, a)=a_{0}$, a point of $S^{2}$. Such a contraction gives rise to a contraction $\tilde{h}:[0,1] \times X_{\infty} \rightarrow \Sigma^{+}$defined by

$$
\left(t, a_{1}, \lambda_{1}\right) \mapsto \tilde{\delta}_{\left(h\left(t, a_{1}\right), \lambda_{1}\right)}+\bar{v}, \quad a_{1} \in X_{1}, \quad \lambda_{1} \geq A .
$$

For $t=0$, we have $\tilde{\delta}_{\left(h\left(0, a_{1}\right), \lambda_{1}\right)}+\bar{v}=\tilde{\delta}_{a_{1}, \lambda_{1}}+\bar{v} \in X_{\infty}$. Furthermore, $\tilde{h}$ is continuous and $\tilde{h}\left(1, a_{1}, \lambda_{1}\right)=\tilde{\delta}_{a_{0}, \lambda_{1}}+\bar{v}$. In addition, using Proposition 2.4, we deduce that

$$
J\left(\tilde{\delta}_{h\left(t, a_{1}\right), \lambda_{1}}+\bar{v}\right) \sim\left(S_{3} / 2\right)^{2 / 3}\left(K\left(h\left(t, a_{1}\right)\right)\right)^{-1 / 3}\left(1+O\left(A^{-2}\right)\right) .
$$

For $c_{0}$ small enough, such a contraction is performed under the level $c_{1}$, for $A$ large enough. So $X_{\infty}$ is contractible in $J_{c_{1}}$, which retracts by deformation onto $X_{\infty}$; thus $X_{\infty}$ is contractible, and so is $X_{1}$, which contradicts assumption $\left(A_{5}\right)$. Hence (1-4) has a solution.

Arguing by contradiction, we may assume that the Morse index of the solution provided by Theorem 1.3 is less than or equal to $m-1$. Perturbing $J$ if necessary, we may assume that all the critical points of $J$ are nondegenerate, have Morse index 
less than or equal to $m-1$, and hence do not change the $m$-dimensional homology group of the level sets of $J$. Since $X_{\infty}$ defines a nontrivial homology class in dimension $m$ but a trivial one in $J_{c_{1}}$, our result follows.

Proof of Theorem 1.4. Under the assumption of the theorem, $\left(y_{0}\right)$ is not a critical point at infinity. Furthermore, for $\mu$ large enough,

$$
\begin{aligned}
\int_{\mathbb{R}_{+}^{3}} K \delta_{\left(y_{0}, \mu\right)}^{6}=K\left(y_{0}\right)\left(S_{3} / 2\right)+ & \int \nabla \\
& \left.\quad+\frac{1}{2} \int y_{0}\right)\left(x-y_{0}\right) \delta_{\left(y_{0}, \mu\right)}^{6} K\left(y_{0}\right)\left(x-y_{0}, x-y_{0}\right) \delta_{\left(y_{0}, \mu\right)}^{6}+o\left(\frac{1}{\mu^{2}}\right) \\
= & K\left(y_{0}\right)\left(S_{3} / 2\right)-\frac{\bar{c}}{\mu} \frac{\partial K}{\partial v}\left(y_{0}\right)+\overline{\bar{c}} \frac{\Delta K\left(y_{0}\right)}{\mu^{2}}+o\left(\frac{1}{\mu^{2}}\right),
\end{aligned}
$$

where $\bar{c}$ and $\overline{\bar{c}}$ are positive constants. Expanding $J$, we obtain

$$
J\left(\tilde{\delta}_{\left(y_{0}, \mu\right)}\right)=\frac{\left(S_{3} / 2\right)^{2 / 3}}{K\left(y_{0}\right)^{1 / 3}}\left(1+\frac{2 S_{3}^{-1}}{3 K\left(y_{0}\right)}\left(\frac{\bar{c}}{\mu} \frac{\partial K}{\partial v}\left(y_{0}\right)-\overline{\bar{c}} \frac{\Delta K\left(y_{0}\right)}{\mu^{2}}\right)+o\left(\frac{1}{\mu^{2}}\right)\right) .
$$

Using the fact that $-\Delta K\left(y_{0}\right)<0$ and $(\partial K / \partial \nu)\left(y_{0}\right)=0$, we derive that

$$
J\left(\tilde{\delta}_{\left(y_{0}, \mu\right)}\right)<c_{\infty}\left(y_{0}\right):=\frac{\left(S_{3} / 2\right)^{2 / 3}}{K\left(y_{0}\right)^{1 / 3}}=\min \left\{c_{\infty}(y): \nabla K_{1}(y)=0\right\} .
$$

Using Proposition 2.4, we see that

$$
J(u) \geq c_{\infty}(a)-c \varepsilon \geq c_{\infty}\left(y_{0}\right)-c \varepsilon
$$

for each $u=\alpha \tilde{\delta}_{(a, \lambda)}+v \in V_{b}(1, \varepsilon)$. Thus, for a fixed $\mu$, we can choose $\varepsilon$ so that

$$
J(u)>J\left(\tilde{\delta}_{\left(y_{0}, \mu\right)}\right) \quad \text { for each } u \in V_{b}(1, \varepsilon) .
$$

Let $u(s)$ be the solution of

$$
\frac{\partial u}{\partial s}=-\nabla J(u), \quad u(0)=\tilde{\delta}_{\left(y_{0}, \mu\right)} .
$$

Observe that (4-1) implies $u(s) \notin V_{b}(p, \varepsilon)$ for $p \geq 1$. Arguing by contradiction, we suppose (1-4) has no solution under the level $c_{\infty}\left(y_{0}\right)$. Thus $|\nabla J(u(s))| \geq c$ for $s \geq 0$ (where $c$ depends only on $\varepsilon$ ). Hence $\frac{\partial}{\partial s} J(u(s))=-|\nabla J(u(s))|^{2} \leq-c^{2}$ for $s \geq 0$. This implies that $J(u(s))$ tends to $-\infty$ as $s$ tends to $+\infty$, which is a contradiction, and so the result follows.

Before providing the proof of Theorem 1.5, we state the following Lemma, the proof of which is very similar to that of [Bahri and Coron 1988, Corollary B.3]; see also [Bahri 1996]. 
Lemma 4.1. Let $a_{1}, a_{2} \in \partial S_{+}^{3}$, let $\alpha_{1}, \alpha_{2}>0$ and choose $\lambda$ sufficiently large. Let $u=\alpha_{1} \tilde{\delta}_{\left(a_{1}, \lambda\right)}+\alpha_{2} \tilde{\delta}_{\left(a_{2}, \lambda\right)}$. Then

$$
J(u) \leq\left(\frac{S_{3}}{2}\left(\frac{1}{\sqrt{K\left(a_{1}\right)}}+\frac{1}{\sqrt{K\left(a_{2}\right)}}\right)\right)^{2 / 3}(1+o(1)):=c_{\infty}\left(a_{1}, a_{2}\right)(1+o(1)) .
$$

Proof of Theorem 1.5. We argue by contradiction. Suppose that $J$ has no critical point in $\Sigma^{+}$, and let

$$
c_{\infty}\left(y_{0}, y_{i_{0}}\right)=\left(\frac{S_{3}}{2}\left(\frac{1}{\sqrt{K\left(y_{0}\right)}}+\frac{1}{\sqrt{K\left(y_{i_{0}}\right)}}\right)\right)^{2 / 3} .
$$

Under assumption (i) of the theorem, using Corollary 3.2, $\left(y_{0}, y_{i_{0}}\right)$ is not a critical point at infinity. By Corollary 3.2, assumption (ii) implies that $\tilde{\delta}_{\left(y_{0}, \infty\right)}$ and $\tilde{\delta}_{\left(y_{0}, \infty\right)}$ are the only critical points at infinity of $J$ under the level $c_{1}=c_{\infty}\left(y_{0}, y_{i_{0}}\right)+\gamma$, for $\gamma$ small enough. The unstable manifolds $W_{u}\left(y_{0}\right)_{\infty}$ and $W_{u}\left(y_{i_{0}}\right)_{\infty}$ of these critical points can be described, by Proposition 2.8, as the product of $W_{s}\left(y_{0}\right)$ and $W_{s}\left(y_{i_{0}}\right)$ (for a pseudogradient of $K$ ) with $[A, \infty$ ) (the domain of the variable $\lambda$ ) for some sufficiently large positive number $A$. Let

$$
X_{2}=\overline{W_{s}\left(y_{i_{0}}\right)} .
$$

Under assumption $\left(A_{6}\right), X_{2}=W_{s}\left(y_{i_{0}}\right) \cup W_{s}\left(y_{0}\right)$ is a compact manifold without boundary, of dimension $k \geq 1$, in $\partial S_{+}^{3}$. Since $J$ has no critical point in $\Sigma^{+}$, it follows that $J_{c_{1}}=\left\{u \in \Sigma^{+}: J(u) \leq c_{1}\right\}$ retracts by deformation onto $X_{\infty}=$ $W_{u}\left(y_{0}\right)_{\infty} \cup W_{u}\left(y_{i_{0}}\right)_{\infty}$ (see [Bahri and Rabinowitz 1991, Sections 7 and 8]) which can be parametrized by $X_{2} \times[A, \infty)$, as already said.

At the same time, $X_{\infty}$ is contractible in $J_{c_{1}}$. Indeed, let

$$
h:[0,1] \times X_{\infty} \rightarrow \Sigma^{+}, \quad(t, x, \lambda) \mapsto \frac{t \tilde{\delta}_{(x, \lambda)}+(1-t) \tilde{\delta}_{\left(y_{0}, \lambda\right)}}{\left\|t \tilde{\delta}_{(x, \lambda)}+(1-t) \tilde{\delta}_{\left(y_{0}, \lambda\right)}\right\|} .
$$

By Lemma 4.1 and the fact that $K(x) \geq K\left(y_{i_{0}}\right)$ for each $x \in X_{2}$, it follows that the contraction $h$ is performed under the level $c_{1}$, so $X_{\infty}$ is contractible in $J_{c_{1}}$ which retracts by deformation onto $X_{\infty}$. Therefore $X_{\infty}$ is contractible, which implies that $X_{2}$ is too, which is a contradiction, since $X_{2}$ is a compact manifold in dimension $k$ without boundary. Hence (1-4) has a solution.

It remains to compute the Morse index of this solution. Using a dimension argument, since $h\left([0,1] \times X_{\infty}\right)$ is a manifold in dimension $k+1$, then the Morse index of the solution provided by Theorem 1.5 is less or equal than $k+1$.

Using the same arguments as those used in the proof of Theorem 1.3, we deduce that the Morse index of the solution provided in Theorem 1.5 is either $k$ or $k+1$. 
Proof of Theorem 1.6. Using Corollary 3.2 and assumption (i) of the theorem, we derive that $\left(y_{0}, y_{i_{0}}\right)$ is not a critical point at infinity. Therefore, we can repeat the proof of Theorem 1.5 without any change, and the result follows.

Proof of Remark 1.7. If we replace assumption (ii) of either of the two preceding theorems by assumption (iii), under the level $c_{1}$, we can find other critical points at infinity but with index not in $\{k, k+1\}$. Using the same arguments as those used in the proofs of Theorems 1.3 and 1.5, Remark 1.7 follows.

Proof of Theorem 1.8. First we notice that $\rho\left(y_{0}, y_{i_{0}}\right)>0$ implies that $\left(y_{0}, y_{i_{0}}\right)_{\infty}$ is a critical point at infinity. Assume that (1-4) has no solution, we claim that $f_{\lambda}\left(C_{y_{0}}(X)\right)$ retracts onto $X_{\infty} \cup W_{u}\left(y_{0}, y_{i_{0}}\right)_{\infty}$. Indeed, let

$$
u=\frac{\alpha K(x)^{-1 / 4} \tilde{\delta}_{(x, \lambda)}+(1-\alpha) K\left(y_{0}\right)^{-1 / 4} \tilde{\delta}_{\left(y_{0}, \lambda\right)}}{\left\|\alpha K(x)^{-1 / 4} \tilde{\delta}_{(x, \lambda)}+(1-\alpha) K\left(y_{0}\right)^{-1 / 4} \tilde{\delta}_{\left(y_{0}, \lambda\right)}\right\|} \in f_{\lambda}\left(C_{y_{0}}(X)\right) .
$$

The action of the flow of the pseudogradient is essentially on $\alpha$. If $\alpha<\frac{1}{2}$, it brings $\alpha$ to zero and thus $u$ approaches $W_{u}\left(y_{0}\right)_{\infty} \equiv\left\{y_{0}\right\}$. If $\alpha>\frac{1}{2}$, the flow brings $\alpha$ to 1 and thus $u$ approaches $W_{u}\left(y_{i_{0}}\right)_{\infty} \equiv X_{\infty}$. If $\alpha=\frac{1}{2}$, then since only $x$ can move, $y_{0}$ remains one of the concentration points of $u$ and $x$ approaches $W_{s}\left(y_{i}\right)$ where $y_{i}$ is either $y_{i_{0}}$ or $y_{0}$ (and only those two cases may occur). If $y_{i}=y_{i_{0}}$, then $u$ goes to $W_{u}\left(y_{0}, y_{i_{0}}\right)_{\infty}$, and if $y_{i}=y_{0}$, then there exists $s_{0}$ such that $x\left(s_{0}\right)$ is close to $y_{0}$. Thus, using Lemma 4.1, we have the estimate

$$
J\left(u\left(s_{0}\right)\right) \leq C_{\infty}\left(y_{0}, y_{0}\right)+\gamma,
$$

for $\gamma$ small enough. Observe that $\left(A_{7}\right)$ implies that $c_{\infty}\left(y_{0}, y_{0}\right)<c_{\infty}(y)$ for each $y \in$ $I_{+} \cup I_{0}^{+} \backslash\left\{y_{0}\right\}$. Thus, using Corollary 3.2, we derive that $J_{c_{\infty}\left(y_{0}, y_{0}\right)+\gamma}$ retracts onto $W_{u}\left(y_{0}\right)_{\infty} \equiv\left\{y_{0}\right\}$ and thus $u$ goes to $W_{u}\left(y_{0}\right)_{\infty}$. Therefore $f_{\lambda}\left(C_{y_{0}}(X)\right)$ retracts onto $X_{\infty} \cup W_{u}\left(y_{0}, y_{i_{0}}\right)_{\infty}$. Since $\mu\left(y_{0}, y_{i_{0}}\right)=0$, it follows that this strong retract does not intersect $W_{u}\left(y_{0}, y_{i_{0}}\right)_{\infty}$ and thus is contained in $X_{\infty}$. Hence $X_{\infty}$ is contractible, and it follows that $X$ is contractible. This yields a contradiction since $X$ is a compact manifold in dimension $k$ without boundary. Hence (1-4) has a solution which, by an argument similar to that in the proof of Theorem 1.3 has index either $k$ or $k+1$.

\section{References}

[Ambrosetti et al. 2000] A. Ambrosetti, Y. Li, and A. Malchiodi, "Scalar curvature under boundary conditions", C. R. Acad. Sci. Paris Sér. I Math. 330:11 (2000), 1013-1018. MR 2001h:53046 Zbl 0969.58005

[Aubin and Bahri 1997] T. Aubin and A. Bahri, "Méthodes de topologie algébrique pour le problème de la courbure scalaire prescrite", J. Math. Pures Appl. (9) 76:6 (1997), 525-549. MR 98g:53068 Zbl 0886.58109 
[Bahri 1989] A. Bahri, Critical points at infinity in some variational problems, Pitman Research Notes in Mathematics Series 182, Longman Scientific \& Technical, Harlow, 1989. MR 91h:58022 Zbl 0676.58021

[Bahri 1996] A. Bahri, "An invariant for Yamabe-type flows with applications to scalar-curvature problems in high dimension”, Duke Math. J. 81:2 (1996), 323-466. MR 97h:53037 Zbl 0856.53028

[Bahri and Coron 1988] A. Bahri and J.-M. Coron, "On a nonlinear elliptic equation involving the critical Sobolev exponent: the effect of the topology of the domain", Comm. Pure Appl. Math. 41:3 (1988), 253-294. MR 89c:35053 Zbl 0649.35033

[Bahri and Coron 1991] A. Bahri and J.-M. Coron, "The scalar-curvature problem on the standard three-dimensional sphere”, J. Funct. Anal. 95:1 (1991), 106-172. MR 92k:58055 Zbl 0722.53032

[Bahri and Rabinowitz 1991] A. Bahri and P. H. Rabinowitz, "Periodic solutions of Hamiltonian systems of 3-body type", Ann. Inst. H. Poincaré Anal. Non Linéaire 8:6 (1991), 561-649. MR 92k:58223 Zbl 0745.34034

[Ben Ayed et al. 1996] M. Ben Ayed, Y. Chen, H. Chtioui, and M. Hammami, "On the prescribed scalar curvature problem on 4-manifolds”, Duke Math. J. 84:3 (1996), 633-677. MR 97g:53047 Zbl 0862.53034

[Ben Ayed et al. 2002] M. Ben Ayed, K. El Mehdi, and M. Ould Ahmedou, "Prescribing the scalar curvature under minimal boundary conditions on the half sphere", Adv. Nonlinear Stud. 2:2 (2002), 93-116. MR 2004b:53050

[Ben Ayed et al. 2005] M. Ben Ayed, K. El Mehdi, and M. Ould Ahmedou, "The scalar curvature problem on the four dimensional half sphere", Calc. Var. Partial Differential Equations 22:4 (2005), 465-482. MR 2124629

[Bianchi and Pan 1999] G. Bianchi and X.-B. Pan, "Yamabe equations on half-spaces", Nonlinear Anal. 37:2, Ser. A: Theory Methods (1999), 161-186. MR 2001b:35103 Zbl 0941.35025

[Brezis and Coron 1985] H. Brezis and J.-M. Coron, "Convergence of solutions of $H$-systems or how to blow bubbles", Arch. Rational Mech. Anal. 89:1 (1985), 21-56. MR 86g:53007 Zbl 0584. 49024

[Chang and Yang 1991] S.-Y. A. Chang and P. C. Yang, "A perturbation result in prescribing scalar curvature on $S^{n}$ ”, Duke Math. J. 64:1 (1991), 27-69. MR 92m:53063 Zbl 0739.53027

[Chang et al. 1993] S.-Y. A. Chang, M. J. Gursky, and P. C. Yang, "The scalar curvature equation on 2- and 3-spheres”, Calc. Var. Partial Differential Equations 1:2 (1993), 205-229. MR 94k:53055 Zbl 0822.35043

[Cherrier 1984] P. Cherrier, "Problèmes de Neumann non linéaires sur les variétés riemanniennes", J. Funct. Anal. 57:2 (1984), 154-206. MR 86c:58154 Zbl 0552.58032

[Chtioui and El Mehdi 2003] H. Chtioui and K. El Mehdi, "Prescribed scalar curvature with minimal boundary mean curvature on $S_{+}^{4}$ ”, preprint IC2003057, ICTP, 2003.

[Djadli et al. 2003] Z. Djadli, A. Malchiodi, and M. Ould Ahmedou, "Prescribing scalar and boundary mean curvature on the three dimensional half sphere", J. Geom. Anal. 13:2 (2003), 255-289. MR 2004d:53032

[Escobar 1996] J. F. Escobar, "Conformal metrics with prescribed mean curvature on the boundary", Calc. Var. Partial Differential Equations 4:6 (1996), 559-592. MR 97h:53040 Zbl 0867.53034

[Han and Li 2000] Z.-C. Han and Y. Li, "The existence of conformal metrics with constant scalar curvature and constant boundary mean curvature", Comm. Anal. Geom. 8:4 (2000), 809-869. MR 2001m:53062 Zbl 0990.53033

[Hebey 1990] E. Hebey, "Changements de métriques conformes sur la sphère. Le problème de Nirenberg”, Bull. Sci. Math. 114:2 (1990), 215-242. MR 91h:53017 Zbl 0713.53023 
[Li 1995a] Y. Li, "The Nirenberg problem in a domain with boundary", Topol. Methods Nonlinear Anal. 6:2 (1995), 309-329. MR 97i:35040 Zbl 0870.35036

[Li 1995b] Y. Y. Li, "Prescribing scalar curvature on $S^{n}$ and related problems. I", J. Differential Equations 120:2 (1995), 319-410. MR 98b:53031 Zbl 0827.53039

[Lions 1985] P.-L. Lions, "The concentration-compactness principle in the calculus of variations. The limit case. II”, Rev. Mat. Iberoamericana 1:2 (1985), 45-121. MR 87j:49012 Zbl 0704.49006

[Milnor 1965] J. Milnor, Lectures on the h-cobordism theorem, Notes by L. Siebenmann and J. Sondow, Princeton University Press, Princeton, N.J., 1965. MR 32 \#8352 Zbl 0161.20302

[Rey 1997] O. Rey, "Boundary effect for an elliptic Neumann problem with critical nonlinearity", Comm. Partial Differential Equations 22:7-8 (1997), 1055-1139. MR 98h:35081 Zbl 0891.35040

[Schoen and Zhang 1996] R. Schoen and D. Zhang, "Prescribed scalar curvature on the $n$-sphere", Calc. Var. Partial Differential Equations 4:1 (1996), 1-25. MR 97j:58027 Zbl 0843.53037

[Struwe 1984] M. Struwe, "A global compactness result for elliptic boundary value problems involving limiting nonlinearities”, Math. Z. 187:4 (1984), 511-517. MR 86k:35046 Zbl 0535.35025

Received August 11, 2003. Revised October 27, 2004.

\author{
Mohamed Ben Ayed \\ DÉPARTEMENT DE MATHÉMATIQUES \\ FACULTÉ DES SCIENCES DE SFAX \\ ROUTE SOUKRA \\ 3018, SFAX \\ TUNISIA \\ Mohamed.Benayed@fss.rnu.tn \\ Hichem Chtioui \\ DÉPARTEMENT DE MATHÉMATIQUes, \\ FACUlté des SCIENCES DE SFAX, \\ ROUTE SOUKRa \\ 3018, SFAX \\ TUNISIA
}

Hichem.Chtioui@fss.rnu.tn 